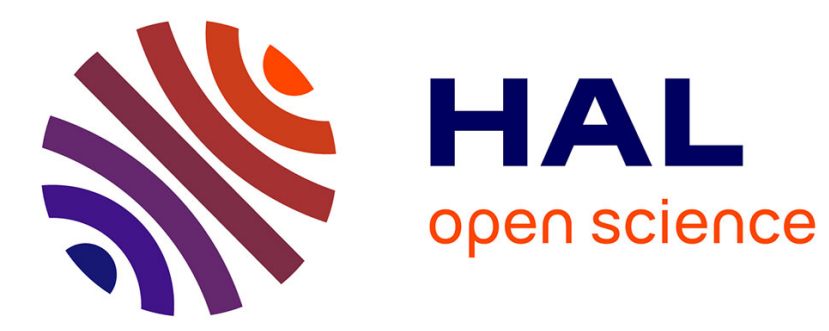

\title{
Un croisement de niveaux singulier : l'anticroisement empêché ou croisement de deuxième espèce
}

\author{
G. Grynberg, J. Dupont-Roc, S. Haroche, C. Cohen-Tannoudji
}

\section{To cite this version:}

G. Grynberg, J. Dupont-Roc, S. Haroche, C. Cohen-Tannoudji. Un croisement de niveaux singulier : l'anticroisement empêché ou croisement de deuxième espèce. Journal de Physique, 1973, 34 (7), pp.523-535. 10.1051/jphys:01973003407052300 . jpa-00207414

\section{HAL Id: jpa-00207414 https://hal.science/jpa-00207414}

Submitted on 1 Jan 1973

HAL is a multi-disciplinary open access archive for the deposit and dissemination of scientific research documents, whether they are published or not. The documents may come from teaching and research institutions in France or abroad, or from public or private research centers.
L'archive ouverte pluridisciplinaire HAL, est destinée au dépôt et à la diffusion de documents scientifiques de niveau recherche, publiés ou non, émanant des établissements d'enseignement et de recherche français ou étrangers, des laboratoires publics ou privés. 


\title{
UN CROISEMENT DE NIVEAUX SINGULIER : L'ANTICROISEMENT EMPÊCHÉ OU CROISEMENT DE DEUXIÈME ESPĖCE
}

\author{
G. GRYNBERG, J. DUPONT-ROC, S. HAROCHE et C. COHEN-TANNOUDJI
}

Laboratoire de Spectroscopie Hertzienne de l'ENS, 24, rue Lhomond, 75005 Paris, France

(Reçu le 28 décembre 1972)

\begin{abstract}
Résumé. - On montre que deux types de croisements de niveaux peuvent apparaître dans un diagramme d'énergie : les croisements de première espèce entre niveaux de symétries différentes, les croisements de deuxième espèce (ou anticroisements empêchés) entre niveaux de même symétrie ou n'ayant pas une symétrie bien définie.

Des propriétés physiques simples permettent de différencier ces deux types de croisements : caractéristiques des résonances observables au voisinage du croisement, transitions non adiabatiques d'un niveau à l'autre lors d'un balayage suffisamment rapide.

On montre enfin que l'existence de croisements de deuxième espèce n'est pas en contradiction avec le théorème de Wigner-Von Neumann.
\end{abstract}

\begin{abstract}
It is shown that 2 types of level crossings may be found in an energy diagram : crossings of the first kind between 2 levels of different symmetries, crossings of the second kind between 2 levels of the same symmetry or having no definite symmetry.

These 2 types of crossings may be distinguished by simple physical properties : characteristics of the corresponding level crossing resonances, non-adiabatic transitions from one level to the other resulting from a sufficiently fast passage.

It is finally shown that the existence of crossings of the second kind is not in contradiction with the Wigner-Von Neumann theorem.
\end{abstract}

1. Introduction. - L'importance des croisements et anticroisements de niveaux en physique atomique est bien connue [1]: si, lorsqu'on fait varier un paramètre $\omega_{0}, 2$ niveaux d'énergie d'un système physique se croisent ou s'anticroisent (c'est-à-dire se repoussent en passant par une distance minimale), il est possible d'obtenir expérimentalement au voisinage de ces points de croisements ou d'anticroisements des signaux résonnants dont l'étude apporte des renseignements intéressants sur le système étudié.

La possibilité d'observer ces résonances dépend crucialement du mode de préparation et de détection utilisé. La plupart du temps, le système est préparé dans un état qui n'est pas stationnaire (c'est-à-dire qui n'est pas état propre du hamiltonien total $\mathcal{H}$ ). Par exemple, l'excitation optique (en raie large) d'un atome possédant un spin nucléaire $I$ le porte dans un état $\left|\mathrm{m}_{I}, \mathrm{~m}_{J}\right\rangle$, où $\mathrm{m}_{J}$ et $\mathrm{m}_{I}$ sont les composantes sur $\mathrm{Oz}$ des moments cinétiques électronique $\mathbf{J}$ et nucléaire I ; par suite du couplage hyperfin $V$ entre I et $\mathbf{J}, \mid \mathrm{m}_{I}, \mathrm{~m}_{J}>$ n'est pas un état stationnaire du système. De manière générale, nous appellerons $\mathscr{H}_{0}$ le hamiltonien approché dont les états propres permettent de décrire simplement l'excitation et la détec- tion du système. Soient $|\mathrm{a}\rangle$ et $\mid \mathrm{b}>2$ états propres de $\mathcal{H}_{0}$ dont les énergies $E_{\mathrm{a}}$ et $E_{\mathrm{b}}$ se croisent pour une valeur $\omega_{0 \mathrm{c}}$ du paramètre $\omega_{0}$ (Fig. 1).

Nous supposons que l'excitation prépare le système, soit dans l'état $|\mathrm{a}\rangle$, soit dans l'état $|\mathrm{b}\rangle$, soit dans une superposition linéaire de $|\mathrm{a}\rangle$ et $|\mathrm{b}\rangle$. Les deux premiers cas correspondent à une excitation diagonale

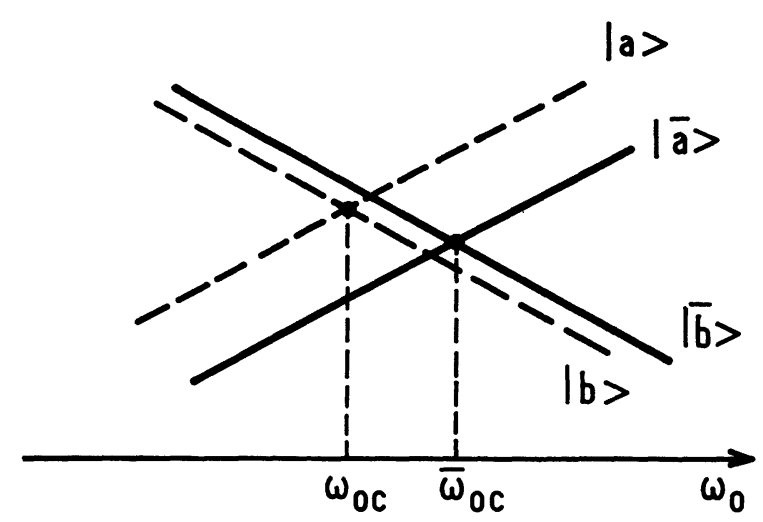

Fig. 1. - Croisement de niveaux (les niveaux non perturbés sont représentés en traits tiretés, les niveaux perturbés en traits pleins). 
dans la base des états propres de $\mathscr{H}_{0}$ (une telle excitation est souvent appelée longitudinale), le troisième à une excitation non diagonale (ou encore transversale). De même, nous supposerons que la détection est sensible soit aux populations des niveaux $\mid a>$ et $|\mathrm{b}\rangle$ (détection diagonale ou encore longitudinale), soit aux éléments non diagonaux $\sigma_{\mathrm{ab}}$ de la matrice densité du système (détection non diagonale ou encore transversale).

Que se passe-t-il lorsqu'on tient compte de la différence $V=\mathscr{H}-H_{0}$ entre le hamiltonien exact $\mathscr{H}$ et le hamiltonien approché $\mathcal{H}_{0}$ ( $V$ est appelé la perturbation) ?

$1.11^{\mathrm{er}}$ CAS : CROISEMENT ENTRE NIVEAUX DE SYMÉTRIES DIFFÉRENTES. - Supposons par exemple que les niveaux $\mid \mathrm{a}>$ et $\mid \mathrm{b}>$ ne soient couplés par $V$ à aucun ordre de perturbation. Dans ce cas, les niveaux perturbés $|\vec{a}\rangle$ et $|\bar{b}\rangle$ continuent en général à se croiser (Fig. 1). Une telle situation est réalisée notamment lorsque, aussi bien les niveaux $\mid \mathrm{a}>$ et $\mid \mathrm{b}>$ que les niveaux $\mid \bar{a}>$ et $\mid \bar{b}>$ sont de symétries différentes : il existe par exemple une observable $L$, associée à une opération de symétrie du système, indépendante $d u$ paramètre $\omega_{0}$ et commutant quel que soit $\omega_{0}$ aussi bien avec $\mathfrak{H}_{0}$ qu'avec $\mathfrak{H}$, les niveaux $|\mathrm{a}\rangle$ et $|\overline{\mathrm{a}}\rangle$ correspondant à une même valeur propre de $L$, distincte de celle qui est associée à $|\mathrm{b}\rangle$ et $|\overline{\mathrm{b}}\rangle$. Ainsi, dans l'exemple cité plus haut, l'invariance du système par rotation autour de la direction $\mathrm{Oz}$ du champ statique $\mathbf{H}_{0}$ entraîne que $F_{\mathrm{z}}=J_{\mathrm{z}}+I_{\mathrm{z}}$ commute quel que soit $\mathbf{H}_{0}$ avec $\mathscr{H}_{0}$ et $H_{F} ; \mathrm{m}_{F}=\mathrm{m}_{J}+\mathrm{m}_{I}$ est alors un bon nombre quantique quel que soit $\mathbf{H}_{0}$, et 2 états $\mid \mathrm{m}_{I}, \mathrm{~m}_{J}>$ et $\left|\mathrm{m}_{I}^{\prime}, \mathrm{m}_{J}^{\prime}\right\rangle$ correspondant à des valeurs $m_{F}$ et $m_{F}^{\prime}$ distinctes ne peuvent jamais être couplés par $V$.

Il est bien connu que l'on peut observer des signaux résonnants au voisinage du point de croisement de la figure 1. L'équation d'évolution de l'élément non diagonal $\sigma_{\mathrm{a}-\bar{b}}^{-}=\langle\overline{\mathrm{a}}|\sigma| \overline{\mathrm{b}}\rangle$ de la matrice densité du système s'écrit en effet :

$$
\frac{\mathrm{d}}{\mathrm{d} t} \sigma_{-\overline{\mathrm{ab}}}=\frac{1}{T_{\mathrm{p}}} \sigma_{\mathrm{ab}}^{(\mathrm{exc})}-i\left(E_{-\mathrm{a}}-E_{-\overline{\mathrm{b}}}\right) \sigma_{-\overline{\mathrm{ab}}}-\Gamma \sigma_{-\overline{\mathrm{ab}}} .
$$

Le premier terme décrivant la préparation (avec une constante de temps $T_{\mathrm{p}}$ ) du système dans une superposition linéaire des états propres $|\bar{a}\rangle$ et $|\bar{b}\rangle$, le second, l'évolution propre due au hamiltonien $\mathscr{H}$, le troisième, l'amortissement du système (avec une constante de temps $1 / \Gamma$ ) dû aux processus de relaxation (émission spontanée, collisions...). La solution en régime stationnaire de (1.1) est évidente :

$$
\sigma_{\mathrm{ab}}^{--}=\frac{\sigma_{-\mathrm{ab}}^{(\mathrm{exc})}}{T_{\mathrm{p}}} \frac{1}{\Gamma+i\left(E_{\mathrm{a}}^{-}-E_{\mathrm{b}}^{-}\right)} .
$$

Ainsi, si la préparation est telle que $\sigma_{-\frac{-}{b}}^{\text {(exc) }}$ est différent de zéro, et si la détection est sensible à l'élément non diagonal $\sigma_{\mathrm{a} \mathrm{b}}^{--}$, on voit qu'on observera une résonance centrée autour du point de croisement $\bar{\omega}_{0 \mathrm{c}}$ de la figure 1, et dont la largeur est déterminée par $\Gamma$ et par la pente relative des deux niveaux perturbés ( $\Gamma$ dans le cas d'un niveau excité est la largeur naturelle du niveau étudié).

Comme nous l'avons vu plus haut, la préparation et la détection du système sont décrites simplement dans la base des états propres de $\mathfrak{H}_{0}$ et il nous faut examiner les conditions auxquelles elles doivent satisfaire pour que la résonance précédente soit observable. Lorsque les niveaux $\mid \mathrm{a}>$ et $|\overline{\mathrm{a}}\rangle$ ont une symétrie différente de celle de $|\mathrm{b}\rangle$ et $|\overline{\mathrm{b}}\rangle$, il est facile de voir que les développements de perturbation de $|\bar{a}\rangle$ et $|\bar{b}\rangle$ ne contiennent aucun état non perturbé commun (puisque $\mid \bar{a}>$ et $\mid \bar{b}>$ se développent sur des états correspondant à des valeurs propres différentes de $L$ ).

En préparant (détectant) le système dans un état propre de $\mathscr{H}_{0}$, c'est-à-dire en utilisant une excitation (détection) longitudinale, on ne pourra donc jamais le préparer (détecter) dans une superposition linéaire des deux niveaux perturbés $|\bar{a}\rangle$ et $|\bar{b}\rangle$, ce qui est indispensable pour l'observation de la résonance de croisement. Les résonances de croisements entre niveaux de symétries différentes ne sont donc observables que si l'on utilise une préparation et une détection transversales. Insistons bien sur le fait que le caractère longitudinal ou transversal de la préparation et de la détection est défini par rapport à la base de $\mathcal{H}_{0}$. C'est uniquement quand les niveaux ont des symétries bien définies que ce caractère demeure également le même dans la base des états propres de $\mathscr{H}$.

$1.22^{\mathrm{e}}$ CAS : ANTICROISEMENT ENTRE NIVEAUX DE MÊME SYMÉTRIE. - Supposons maintenant que les 2 niveaux $|\mathrm{a}\rangle$ et $|\mathrm{b}\rangle$ de la figure 1 soient couplés par $V: V_{\mathrm{ab}}=\langle\mathrm{a}|V| \mathrm{b}\rangle \neq 0$. Dans l'exemple cité plus haut, c'est ce qui se passe pour 2 niveaux $\left|\mathrm{m}_{I}, \mathrm{~m}_{J}\right\rangle$ correspondant à la même valeur de $\mathrm{m}_{F}$, c'est-à-dire de même symétrie.

On trouve alors que les 2 niveaux perturbés $|\bar{a} \bar{b}\rangle+$ et $|\overline{\mathrm{ab}}\rangle_{\text {- }}$ auxquels ils donnent naissance ne se
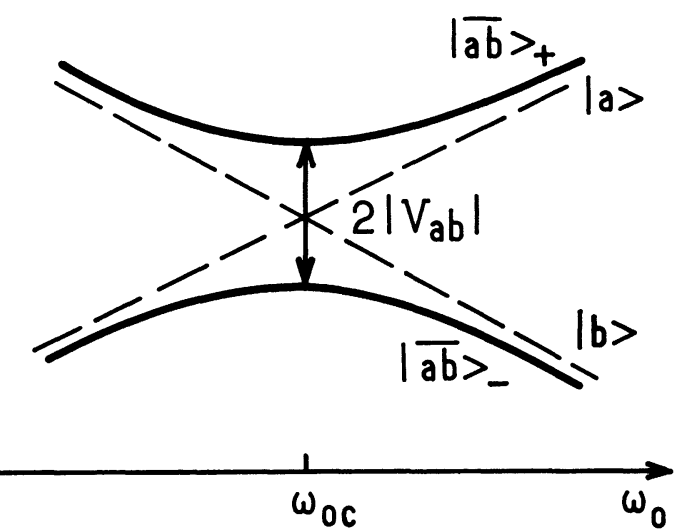

Fig. 2. - Anticroisement de niveaux : sous l'effet du couplage $V_{\mathrm{ab}}$, les niveaux non perturbés (représentés en traits tiretés) donnent naissance à deux niveaux perturbés (représentés en traits pleins) qui passent à une distance minimale l'un de l'autre lorsqu'on fait varier $\omega_{0}$ 
croisent plus mais se repoussent mutuellement lorsqu'on balaie $\omega_{0}$ autour de $\omega_{0 c}$ (Fig. 2 ); ils passent à une distance minimale l'un de l'autre égale à $2\left|V_{\mathrm{ab}}\right|$ puis tendent asymptotiquement vers les niveaux non perturbés, formant ce qu'on appelle un anticroisement. Par ailleurs, les états propres $|\overline{\mathrm{ab}}\rangle_{ \pm}$de $\mathcal{H}$ sont des combinaisons linéaires de $|\mathrm{a}\rangle$ et $|\vec{b}\rangle$ qui varient rapidement autour de $\omega_{0}=\omega_{0 c}$. Connaissant ces états propres et les énergies correspondantes, on peut calculer aisément la probabilité pour que, étant préparé dans l'état non stationnaire $|\mathrm{a}\rangle$ à l'instant $t=0$, le système soit trouvé à l'instant $t$ dans l'état non stationnaire $|\mathrm{b}\rangle$. Un calcul très simple donne la formule de Rabi :

$$
\begin{aligned}
P_{\mathrm{a} \rightarrow \mathrm{b}}(t) & =\frac{4\left|V_{\mathrm{ab}}\right|^{2}}{4\left|V_{\mathrm{ab}}\right|^{2}+\left(E_{\mathrm{a}}-E_{\mathrm{b}}\right)^{2}} \times \\
& \times \sin ^{2}\left[\sqrt{4\left|V_{\mathrm{ab}}\right|^{2}+\left(E_{\mathrm{a}}-E_{\mathrm{b}}\right)^{2}} \frac{t}{2 \hbar}\right] .
\end{aligned}
$$

Lorsqu'on tient compte de la dispersion des temps $t$ due aux processus de relaxation caractérisés par $\Gamma$, on peut introduire une probabilité de transition moyenne $\bar{P}_{\mathrm{a} \rightarrow \mathrm{b}}$ définie par

$$
\begin{aligned}
\bar{P}_{\mathrm{a} \rightarrow \mathrm{b}} & =\Gamma \int_{0}^{\infty} \mathrm{e}^{-\Gamma t} P_{\mathrm{a} \rightarrow \mathrm{b}}(t) \mathrm{d} t \\
& =\frac{2\left|V_{\mathrm{ab}}\right|^{2}}{\Gamma^{2}+4\left|V_{\mathrm{ab}}\right|^{2}+\left(E_{\mathrm{a}}-E_{\mathrm{b}}\right)^{2}}
\end{aligned}
$$

Les variations résonnantes de $\bar{P}_{\mathrm{a} \rightarrow \mathrm{b}}$ lorsqu'on balaie $\omega_{0}$ autour de $\omega_{0 \mathrm{c}}$ reflètent donc un transfert résonnant de population entre les états propres non perturbés. Elles peuvent être détectées expérimentalement si l'on prépare le système dans l'état $|\mathrm{a}\rangle$ et qu'on le détecte dans l'état $|\mathrm{b}\rangle$, c'est-à-dire si l'on utilise une préparation et une détection longitudinales. On note là une première différence avec les résonances décrites au paragraphe précédent qui nécessitaient une préparation et une détection transversales. De plus, on voit sur les expressions (1.2) et (1.4) que, lorsque $\Gamma \rightarrow 0$, la largeur des résonances de croisements tend vers zéro, alors que celle des résonances d'anticroisements tend vers une quantité proportionnelle à $\left|V_{\mathrm{ab}}\right|$. Il s'agit là d'une deuxième différence importante entre les deux types de résonances.

Notons enfin qu'il n'est pas nécessaire que $\left|V_{\mathrm{ab}}\right|$ soit différent de zéro pour que les deux niveaux s'anticroisent. $V$ peut coupler $|\mathrm{a}\rangle$ et $|\mathrm{b}\rangle$, non pas directement $\left(V_{\mathrm{ab}}=0\right)$, mais indirectement, par l'intermédiaire d'autres états non perturbés $|\mathrm{i}\rangle, \mid \mathrm{j}>\ldots$ Par exemple, un tel couplage indirect au second ordre existe lorsque la quantité

$$
\left.R_{\mathrm{ab}}\left(E_{0}\right)=\sum_{\mathrm{i} \neq \mathrm{a}, \mathrm{b}}<\mathrm{a}|V| \mathrm{i}>\frac{1}{E_{0}-E_{\mathrm{i}}}<\mathrm{i}|V| \mathrm{b}\right\rangle
$$

(où $E_{0}$ est l'énergie commune des 2 niveaux $|\mathrm{a}\rangle$ et $|\mathrm{b}\rangle$ au point de croisement) est non nulle. La formule (1.4) demeure valable à condition de remplacer $V_{\mathrm{ab}}$ par $R_{\mathrm{ab}}\left(E_{0}\right)\left(^{1}\right)$. Les dénominateurs d'énergie $1 /\left(E_{0}-E_{\mathrm{i}}\right)$ qui figurent dans (1.5) expriment que le couplage'indirect entre $\mid \mathrm{a}>$ et $\mid \mathrm{b}>$ est d'autant plus petit que les états intermédiaires $\mid \mathrm{i}>$ sont plus éloignés des états $\mid \mathrm{a}>$ et $\mid \mathrm{b}>$. Lorsque $V_{\mathrm{ab}}$ est nul mais que $R_{\mathrm{ab}}\left(E_{0}\right)$ ne l'est pas, on dit que l'anticroisement est d'un ordre supérieur.

$1.33^{\mathrm{e}}$ CAS : CROISEMENTS DE DEUXIÈME ESPECE. Supposons enfin que les 2 niveaux $|a\rangle$ et $|\mathrm{b}\rangle$ ne soient pas couplés directement, mais qu'il existe entre eux un couplage indirect s'annulant précisément au point de croisement non perturbé :

$$
\begin{gathered}
V_{\mathrm{ba}}=0 \\
R_{\mathrm{ba}}(E)\left\{\begin{array}{lll}
=0 & \text { si } & E=E_{0} \\
\neq 0 & \text { si } & E \neq E_{0}
\end{array}\right.
\end{gathered}
$$

On peut par exemple imaginer qu'il existe plusieurs états intermédiaires permettant de relier $|\mathrm{a}\rangle$ et $|\mathrm{b}\rangle$ au second ordre, chacun des termes correspondants de $R_{\mathrm{ba}}(E)$ étant visualisé par un diagramme sur la figure 3 :

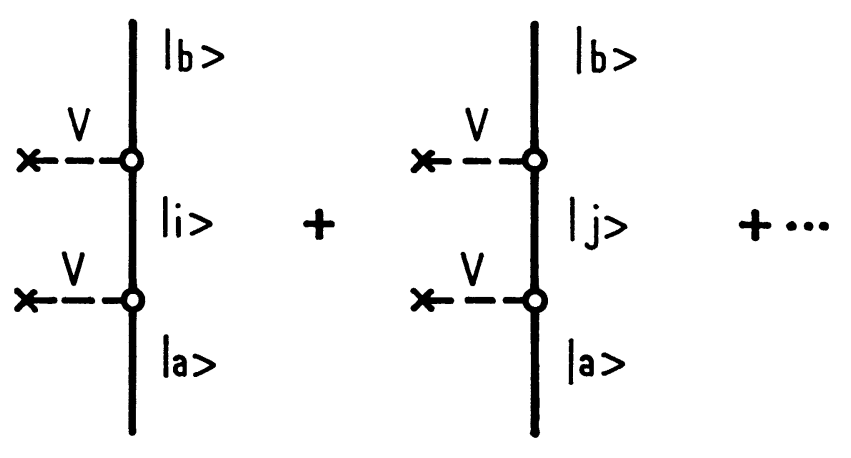

FIG. 3. - Représentation diagrammatique d'un couplage indirect entre les états $|a|$ et $|\mathrm{b}\rangle$, via les états intermédiaires $|\mathrm{i}\rangle, \mid \mathrm{j}>\ldots$ Pour un croisement de deuxième espèce, les diverses amplitudes correspondantes interfèrent destructivement au point de croisement (on a de plus $\langle\mathrm{b}|\mathrm{V}| \mathrm{a}\rangle=0$ ).

La situation que nous envisageons ici est celle où tous les diagrammes de la figure 3 interfèrent destructivement au point de croisement non perturbé. Nous verrons alors que les 2 niveaux perturbés $\mid \bar{a}>$ et $\mid \bar{b}>$ issus de $|\mathrm{a}\rangle$ et $|\mathrm{b}\rangle$ continuent à se croiser. Il est cependant évident qu'un tel croisement, que nous appellerons croisement de deuxième espèce, doit avoir

(1) Nous avons supposé implicitement que $V_{\mathrm{aa}}$ et $V_{\mathrm{bb}}$ sont nuls (s'ils ne le sont pas, on peut toujours les réintégrer dans $\left.J_{0}\right)$. Lorsque $R_{\mathrm{aa}}\left(E_{0}\right)$ et $R_{\mathrm{bb}}\left(E_{0}\right)$ sont non nuls, ils représentent un déplacement des états $|\mathrm{a}\rangle$ et $|\mathrm{b}\rangle$ dû au couplage non résonnant des états $|\mathrm{a}\rangle$ et $|\mathrm{b}\rangle$ avec les autres états $|\mathrm{i}\rangle$. On peut montrer qu'il en résulte un déplacement des résonances étudiées dans ce paragraphe. 
des propriétés très différentes de celles des croisements étudiés plus haut et que nous appellerons de première espèce, où les 2 niveaux non perturbés $|\mathrm{a}\rangle$ et $|\mathrm{b}\rangle$ ne sont reliés entre eux à aucun ordre, non seulement au point de croisement non perturbé, mais pour tout $\omega_{0}$. On peut en quelque sorte considérer qu'un croisement de deuxième espèce est un anticroisement empêché, et il est par suite normal, comme nous le montrerons effectivement, que les diverses résonances observables au voisinage de ces points aient des caractéristiques intermédiaires entre celles des résonances de croisements de première espèce et celles des résonances d'anticroisements.

1.4 But DE CET ARTICLE. - Le but de cet article est de présenter une étude détaillée des croisements de deuxième espèce et d'en donner un certain nombre d'exemples physiques. Pour bien comprendre les phénomènes qui apparaissent, nous commençons dans le paragraphe 2 par présenter un modèle à 4 niveaux couplés par une perturbation $V$ telle que les conditions (1.6) soient remplies. Les calculs peuvent être menés jusqu'au bout et mettent en évidence les caractéristiques essentielles des croisements de deuxième espèce.

Il nous a paru ensuite important de caractériser ces croisements en partant directement du hamiltonien total $\mathscr{H}$, sans qu'il soit nécessaire d'utiliser la théorie des perturbations en mettant $\mathscr{H}$ sous la forme $\mathscr{H}=\mathscr{H}_{0}+V$. Si en effet un croisement de deuxième espèce pour un découpage $\mathscr{H}=H_{0}+V$ devenait de première espèce pour un autre découpage $J=\mathfrak{H}_{0}^{\prime}+V^{\prime}$, la distinction entre les 2 types de croisements n'aurait plus aucun intérêt. Nous montrons dans le paragraphe 3 de cet article qu'un croisement de deuxième espèce entre 2 états propres $|\bar{a}\rangle$ et $\mid \bar{b}>$ de $\mathcal{H}$ peut également être défini de la manière suivante : le système étant dans l'état $\mid \bar{a}>$ pour la valeur $\omega_{0 \mathrm{i}}$ du paramètre $\omega_{0}$, balayons ce paramètre jusqu'à $\omega_{0 f}$ de manière à traverser le point de croisement $\omega_{0 c}$ (Fig. 4).

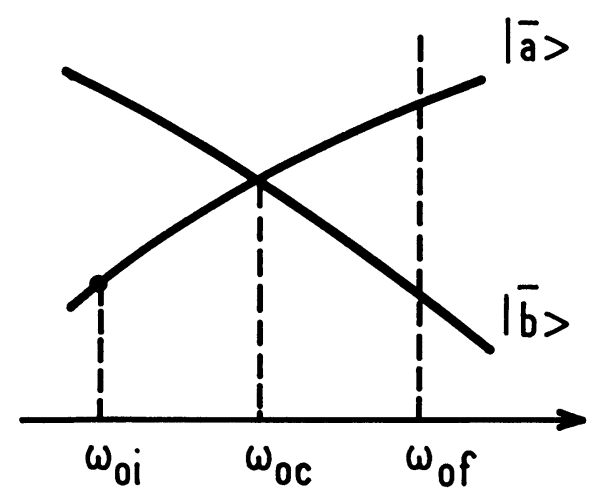

FIG. 4. - Le système étant initialement dans l'état $|\bar{a}\rangle$ pour la valeur $\omega_{0 \mathrm{i}}$ du paramètre $\omega_{0}$, on balaie $\omega_{0}$ de $\omega_{0 \mathrm{i}}$ à $\omega_{0 \text { f. }}$ Si le croisement est de première espèce, le système reste toujours dans l'état $|\bar{a}\rangle$, alors qu'il peut passer dans l'état $|\bar{b}\rangle$ si le croisement est de deuxième espèce.
Alors que pour un croisement de première espèce, le système ne peut jamais passer dans l'état $|\bar{b}\rangle$, quelle que soit la vitesse de balayage, pour un croisement de deuxième espèce, un tel passage est possible si l'on balaie $\omega_{0}$ suffisamment vite.

Cette nouvelle approche non perturbative nous permettra également au paragraphe 4 de préciser la portée du théorème de Von Neumann et Wigner [2] : l'énoncé qui est généralement donné de ce théorème laisse penser que, seuls, les croisements entre niveaux de symétrie différente et indépendante de $\omega_{0}$ (donc de première espèce) peuvent exister. En reprenant la démonstration de ce théorème, nous montrons qu'elle n'exclut pas l'existence de croisements de deuxième espèce entre niveaux de même symétrie ou n'ayant pas une symétrie bien définie.

Nous montrerons enfin dans un second article que les idées précédentes permettent de classer très simplement les divers croisements de niveaux apparaissant dans le diagramme d'énergie d'atomes habillés par des photons de radiofréquence [3]. Nous verrons ainsi que tous les croisements de première espèce sont liés à des symétries bien définies du hamiltonien du système global atome + radiofréquence, symétries se conservant pour tout $\omega_{0}$ alors que les croisements de deuxième espèce sont de natures plus diverses ; certains étant liés au théorème de Majorana, d'autres à l'apparition d'une symétrie accidentelle du hamiltonien pour certaines valeurs du champ statique. Nous présenterons également quelques expériences réalisées sur l'état fondamental de l'isotope ${ }^{201} \mathrm{Hg}$ et destinées à mettre en évidence certains caractères des croisements de deuxième espèce.

2. Etude d'un modèle simple. - 2.1 Нy ET NOTATIONS. - Nous considérons un hamiltonien $\mathscr{H}_{0}$ ayant 4 états propres $|\mathrm{a}\rangle,|\mathrm{b}\rangle,|\mathrm{i}\rangle,|\mathrm{j}\rangle$ d'énergies respectivement égales à $\omega_{0},-\omega_{0}, \omega$ et $-\omega$ ( $\omega$ étant une constante indépendante du paramètre de balayage $\omega_{0}$ ). Les niveaux correspondants sont représentés en traits tiretés sur la figure 5. Quant à la perturbation $V$, nous supposons qu'elle est non diagonale et qu'elle ne relie que les états $|a\rangle$ et $|i\rangle,|a\rangle$ et $|\mathrm{j}\rangle,|\mathrm{b}\rangle$ et $|\mathrm{i}\rangle,|\mathrm{b}\rangle$ et $|\mathrm{j}\rangle$, les éléments de matrice correspondants étant tous égaux à une constante réelle $v$.

Le couplage entre $|\mathrm{a}\rangle$ et $|\mathrm{b}\rangle$ ne peut donc se faire que par l'intermédiaire de $|\mathrm{i}\rangle$ et $|\mathrm{j}\rangle$ et la quantité $R_{\mathrm{ba}}(E)$ qui décrit un tel couplage est donnée par :

$$
\begin{aligned}
R_{\mathrm{ba}}(E) & =\langle\mathrm{b}|\left[V+V \frac{Q}{E-H_{0}} V+\right. \\
& \left.+V \frac{Q}{E-H_{0}} V \frac{Q}{E-H_{0}} V+\cdots\right]|\mathrm{a}\rangle
\end{aligned}
$$

où $Q$ est le projecteur sur le sous-espace sous-tendu $\operatorname{par}|\mathrm{i}\rangle$ et $|\mathrm{j}\rangle$. D'après les hypothèses faites sur $V$, 


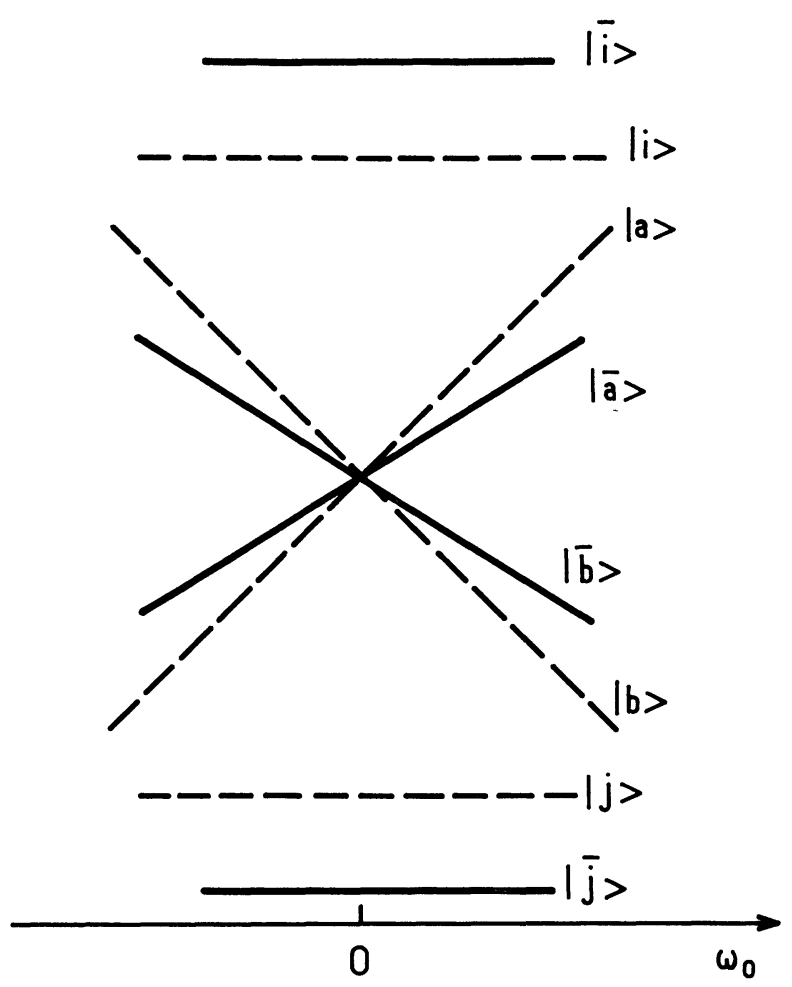

Fig. 5. - Variations avec $\omega_{0}$ des énergies des niveaux non perturbés (traits tiretés) et des niveaux perturbés (traits pleins), pour le modèle simple étudié au paragraphe 2 . Le croisement apparaissant en $\omega_{0}=0$ est un croisement de deuxième espèce.

seul le terme d'ordre 2 de ce développement est non nul et l'on a :

$$
R_{\mathrm{ba}}(E)=\frac{v^{2}}{E-\omega}+\frac{v^{2}}{E+\omega}=\frac{2 E v^{2}}{E^{2}-\omega^{2}} .
$$

On constate que $R_{\mathrm{ba}}(E)$ est nul au point de croisement $(E=0)$, tout en devenant différent de zéro dès qu'on s'écarte de ce point; une telle situation est caractéristique d'un croisement de deuxième espèce.

2.2 Etats PRopres et VAleurs Propres DU HAMILTONIEN TOTAL. - Il est possible de diagonaliser exactement la matrice représentant le hamiltonien total $\mathscr{H}=\mathscr{H}_{0}+V$. Soient $\pm \omega^{\prime}$ et $\pm \omega_{0}^{\prime}$ les valeurs propres de $\mathscr{H}$ qui tendent vers $\pm \omega$ et $\pm \omega_{0}$ lorsque $v \rightarrow 0$. Comme dans la suite, on se placera au voisinage du point de croisement (c'est-à-dire de $\omega_{0}=0$ ), nous nous contenterons de donner ici l'expression de $\omega^{\prime}$ et $\omega_{0}^{\prime}$ lorsque $\omega_{0} / \omega \ll 1$. On trouve ainsi, à l'ordre le plus bas en $\omega_{0} / \omega$ :

$$
\begin{aligned}
& \omega^{\prime 2}=\omega^{2}\left[1+\frac{4 v^{2}}{\omega^{2}}\right] \\
& \omega_{0}^{\prime 2}=\frac{\omega_{0}^{2}}{1+\frac{4 v^{2}}{\omega^{2}}} .
\end{aligned}
$$

Les niveaux perturbés $|\bar{a}\rangle$ et $|\bar{b}\rangle$, issus de $|a\rangle$ et $|\mathrm{b}\rangle$ (et représentés sur la figure 5 en traits pleins de même que les niveaux $|\bar{i}\rangle$ et $|\bar{j}\rangle$ ) continuent donc de se croiser. On constate que leur pente diminue quand le couplage $v$ augmente.

Un tel résultat peut paraître surprenant : comme les 2 niveaux $|\mathrm{a}\rangle$ et $|\mathrm{b}\rangle$ sont couplés en dehors $\mathrm{du}$ point de croisement, on pourrait s'attendre à ce qu'ils se repoussent et acquièrent ainsi une pente plus grande. En fait, pour $\omega_{0}$ non nul, les 2 niveaux $|a\rangle$ et $|\mathrm{b}\rangle$ ne sont couplés entre eux qu'au second ordre et l'effet prépondérant est dû au couplage (au premier ordre) avec les niveaux $|\mathrm{i}\rangle$ et $|\mathrm{j}\rangle$. Pour $\omega_{0}>0$, le niveau $|\mathrm{a}\rangle$ est plus proche du niveau $\mid \mathrm{i}>$ que du niveau $|\mathrm{j}\rangle$ et la répulsion (vers le bas) due au niveau $\mid \mathrm{i}>$ l'emporte sur celle due au niveau $\mid \mathrm{j}>$ (et qui s'exerce vers le haut); le déplacement global du niveau $\mid$ a $>$ est donc négatif et sa pente est diminuée. Quand $\omega_{0}$ tend vers zéro, les répulsions dues à $|\mathrm{i}\rangle$ et $|\mathrm{j}\rangle$ s'équilibrent de mieux en mieux; d'autre part, le couplage indirect avec $|\mathrm{b}\rangle$ tend vers zéro et l'on comprend que les niveaux perturbés continuent à se croiser.

Il est intéressant également d'examiner le comportement des états propres. Si l'on appelle $|\bar{a}\rangle,|\bar{b}\rangle$, $|\overline{\mathrm{i}}\rangle,|\overline{\mathrm{j}}\rangle$ les états propres de $\mathscr{H}$ issus de $|\mathrm{a}\rangle$, $|\mathrm{b}\rangle,|\mathrm{i}\rangle,|\mathrm{j}\rangle$, on obtient, en développant les expressions exactes à l'ordre le plus bas en $\omega_{0} / \omega$ et au second ordre en $v / \omega$ :

$$
\left\{\begin{array}{l}
|\overline{\mathrm{a}}>=| \mathrm{a}>-\frac{v^{2}}{\omega^{2}}\left|\mathrm{~b}>-\frac{v}{\omega}\right| \mathrm{i}>+\frac{v}{\omega} \mid \mathrm{j}> \\
\left|\overline{\mathrm{b}}>=-\frac{v^{2}}{\omega^{2}}\right| \mathrm{a}>+\left|\mathrm{b}>-\frac{v}{\omega}\right| \mathrm{i}>+\frac{v}{\omega} \mid \mathrm{j}> \\
\left|\overline{\mathrm{i}}>=\frac{v}{\omega}\right| \mathrm{a}>+\frac{v}{\omega}|\mathrm{b}>+| \mathrm{i}>+\frac{v^{2}}{\omega^{2}} \mid \mathrm{j}> \\
|\overline{\mathrm{j}}\rangle=-\frac{v}{\omega}\left|\mathrm{a}>-\frac{v}{\omega}\right| \mathrm{b}>+\frac{v^{2}}{\omega^{2}}|\mathrm{i}>+| \mathrm{j}>.
\end{array}\right.
$$

On constate que $\omega_{0}$ a complètement disparu de ces expressions : à la différence de ce qui se passe pour un anticroisement, les fonctions d'ondes perturbées varient donc très peu lorsqu'on balaie $\omega_{0}$. D'autre part, on note que, même au point de croisement, l'état $|\bar{a}\rangle$ est contaminé par l'état $|\mathrm{b}\rangle$ (et l'état $|\bar{b}\rangle$ par l'état $|a\rangle)$, ce qui est exclu pour un croisement de première espèce.

Ces 2 points peuvent se comprendre comme plus haut, en examinant ce qui se passe en dehors du point de croisement, et en faisant tendre ensuite $\omega_{0}$ vers zéro. Pour $\omega_{0} \neq 0$, le développement de perturbation de $|\bar{a}\rangle$ s'obtient en appliquant la théorie des perturbations dans le cas non dégénéré : la contamination de l'état $|\bar{a}\rangle$ par l'état $|\mathrm{b}\rangle$ est proportionnelle au couplage indirect $R_{\mathrm{ab}}\left(E=\omega_{0}\right)$ entre $|\mathrm{a}\rangle$ et $|\mathrm{b}\rangle$ (évalué pour une valeur de $E$ égale à l'énergie non 
perturbée de $|\mathrm{a}\rangle$, c'est-à-dire à $\left.\omega_{0}\right)$, et inversement proportionnelle à l'écart $2 \omega_{0}$ entre les 2 états $|\mathrm{a}\rangle$ et $|\mathrm{b}\rangle$. Comme $R_{\mathrm{ab}}\left(\omega_{0}\right)$ est, d'après (II.2), proportionnel à $\omega_{0}$ (pour $\omega_{0}$ faible), on comprend que le coefficient de contamination, $R_{\mathrm{ab}}\left(\omega_{0}\right) / 2 \omega_{0}$ dépende peu de $\omega_{0}$ et garde la même valeur pour $\omega_{0}=0$.

2.3 RÉSONANCES OBSERVABLES AU VOISINAGE D'UN CROISEMENT DE DEUXIÈME ESPÈCE. - Le fait que l'état $\mid \bar{a}>$ soit contaminé par l'état $\mid \mathrm{b}>$ et l'état $\mid \bar{b}>$ par l'état $\mid \mathrm{a}>$ va nous permettre maintenant de comprendre comment il est possible, en utilisant une excitation diagonale, d'observer une résonance de croisement au voisinage de $\omega_{0}=0$.

Supposons en effet que, par un procédé quelconque, on crée le système dans l'état non perturbé $|\mathrm{a}\rangle$. Cet état n'est pas un état stationnaire. En inversant le système (2.4), on obtient :

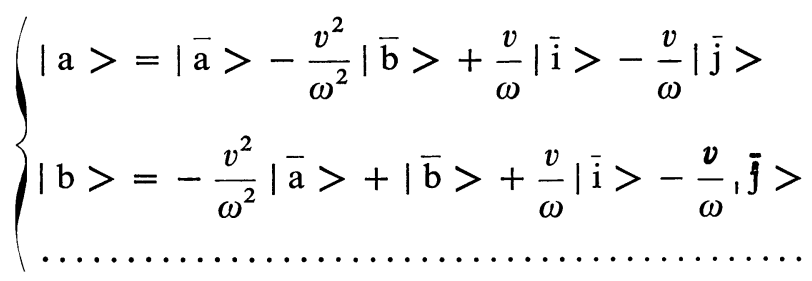

En préparant le système dans l'état $|\mathrm{a}\rangle$, on le porte donc dans une superposition linéaire des 2 états perturbés $|\bar{a}\rangle$ et $|\bar{b}\rangle$ qui se croisent. L'état d'un système préparé à l'instant $t=0$ dans l'état $|\mathrm{a}\rangle$ devient à l'instant $t$ :

$$
\begin{aligned}
\left|\Psi(t)>=\mathrm{e}^{-\mathrm{i} \omega_{0}^{\prime} t}\right| \overline{\mathrm{a}}>-\frac{v^{2}}{\omega^{2}} \mathrm{e}^{\mathrm{i} \omega_{0}^{\prime} t} \mid \overline{\mathrm{b}}>+ \\
\quad+\frac{v}{\omega} \mathrm{e}^{-\mathrm{i} \omega^{\prime} t}\left|\overline{\mathrm{i}}>-\frac{v}{\omega} \mathrm{e}^{\mathrm{i} \omega^{\prime} t}\right| \overline{\mathrm{j}}>
\end{aligned}
$$

L'amplitude de probabilité de trouver le système dans l'état $|\mathrm{b}\rangle$ est alors, d'après (2.5) :

$$
<\mathrm{b} \mid \Psi(t)>=\frac{2 v^{2}}{\omega^{2}}\left(\cos \omega^{\prime} t-\cos \omega_{0}^{\prime} t\right)
$$

ce qui permet de calculer la probabilité de transfert $P_{\mathrm{a} \rightarrow \mathrm{b}}(t):$

$$
\bar{P}_{\mathrm{a} \rightarrow \mathrm{b}}(t)=\frac{4 v^{4}}{\omega^{4}}\left(\cos \omega^{\prime} t-\cos \omega_{0}^{\prime} t\right)^{2} .
$$

Si l'on suppose que le système a une durée de vie finie $1 / \Gamma$ et qu'on le prépare continuellement dans l'état non perturbé $|\mathrm{a}\rangle$, la population du niveau $\mid \mathrm{b}>$ sera, en régime stationnaire, proportionnelle à :

$$
\bar{P}_{\mathrm{a} \rightarrow \mathrm{b}}=\Gamma \int_{0}^{\infty} \mathrm{e}^{-\Gamma t} P_{\mathrm{a} \rightarrow \mathrm{b}}(t) \mathrm{d} t
$$

soit :

$$
\bar{P}_{\mathrm{a} \rightarrow \mathrm{b}}=4 \frac{v^{4}}{\omega^{4}}+2 \frac{v^{4}}{\omega^{4}} \frac{\Gamma^{2}}{\Gamma^{2}+4 \omega_{0}^{\prime 2}} .
$$

Les termes suivants de $\bar{P}_{\mathrm{a} \rightarrow \mathrm{b}}$ sont, soit d'un ordre supérieur, soit non résonnants. En remplaçant $\omega_{0}^{\prime 2}$ par $\omega_{0}^{2} /\left(1+4 v^{2} / \omega^{2}\right)$ et en ne conservant que les termes résonnants à l'ordre le plus bas, on obtient finalement :

$$
\bar{P}_{\mathrm{a} \rightarrow \mathrm{b}}=2 \frac{v^{4}}{\omega^{4}} \frac{\Gamma^{2}}{\Gamma^{2}\left(1+\frac{4 v^{2}}{\omega^{2}}\right)+4 \omega_{0}^{2}}
$$

Nous constatons que $P_{\mathrm{a} \rightarrow \mathrm{b}}$ subit des variations résonnantes lorsqu'on balaie $\omega_{0}$ autour de zéro, sur un intervalle dont la largeur est proportionnelle à $\Gamma$. Les résonances observables au voisinage d'un croisement de deuxième espèce ont donc des caractéristiques qui les apparentent à la fois aux résonances d'anticroisements et aux résonances de croisements de première espèce décrites dans le paragraphe 1 .

(i) Comme des résonances d'anticroisements, elles apparaissent avec une excitation et une détection diagonales et correspondent donc à un transfert résonnant de populations entre les niveaux non perturbés $|a\rangle$ et $|\mathrm{b}\rangle$.

(ii) Par contre, comme les résonances de croisements, la largeur de la résonance tend vers zéro si la largeur naturelle $\Gamma$ des niveaux tend vers zéro. On peut dire encore que la résonance n'est pas due à une variation rapide des fonctions d'onde perturbées au voisinage de $\omega_{0}=0$ (comme c'est le cas pour les résonances d'anticroisements), mais au fait que les 2 niveaux perturbés se croisent : la cohérence hertzienne entre les niveaux $|\bar{a}\rangle$ et $|\bar{b}\rangle$ est en effet introduite par l'excitation avec une efficacité indépendante de $\omega_{0}$; elle évolue à la fréquence $\left(E_{\overline{\mathrm{a}}}-E_{\overline{\mathrm{b}}}\right) / h$ et ne peut s'accumuler de manière importante que lorsque $E_{\overline{\mathrm{a}}}=E_{\overline{\mathrm{b}}}$.

La différence importante entre croisements de première espèce et croisements de deuxième espèce est qu'une excitation diagonale dans la base $\{|\mathrm{a}\rangle,|\mathrm{b}\rangle\}$ reste diagonale dans la base $\{|\bar{a}>,| \bar{b}\rangle\}$ pour un croisement de première espèce, alors qu'elle peut devenir non diagonale dans la base perturbée pour un croisement de deuxième espèce.

3. Définition plus générale des croisements de première et de deuxième espèce. - 3.1 INTRODUCTION. Le traitement de la partie précédente pourrait suggérer que la différence entre croisements de première et de deuxième espèce est uniquement liée à la séparation du hamiltonien $\mathcal{H}$ en un hamiltonien non perturbé $\mathfrak{H}_{0}$ et une perturbation $V$. Nous allons montrer dans cette partie qu'on peut s'affranchir de cette restriction et caractériser l'espèce d'un croisement directement à partir du hamiltonien total $\mathcal{H}$ du système. 
L'idée physique qui va nous guider est la suivante : supposons que le système se trouve à l'instant initial dans l'état $\mid \overline{\mathbf{a}}\left(\omega_{0 \mathrm{i}}\right)>$ pour la valeur $\omega_{0 \mathrm{i}}$ du paramètre $\omega_{0}$ (cf. Fig. 4) et balayons $\omega_{0}$ de $\omega_{0 \mathrm{i}}$ à $\omega_{0 \mathrm{f}}$ pendant le temps $T$ : le système peut-il passer de $|\bar{a}\rangle$ vers $|\bar{b}\rangle$ au cours de cette opération? Nous allons voir que la réponse à cette question n'est pas la même suivant le type de croisement envisagé, ce qui nous permettra de donner une nouvelle définition des croisements de deuxième espèce.

3.2 CRoisementS De PRemière esPèce. - Les croisements de première espèce ont été définis dans la première partie comme des croisements entre des niveaux de symétries différentes. Il existe alors un opérateur hermitique $L$ indépendant du paramètre $\omega_{0}$ et qui commute avec $\Re$. L'équation de Schrödinger, qui régit l'évolution du vecteur d'état lorsqu'on balaie $\omega_{0}$ de $\omega_{0 \mathrm{i}}$ à $\omega_{0 \mathrm{f}}$, se résout indépendamment dans les divers sous-espaces propres de $L:$ le système ne peut transiter que vers des états ayant les mêmes propriétés de symétrie que $|\bar{a}\rangle$. Si le croisement est de première espèce, la probabilité de transition de $|\bar{a}\rangle$ vers $|\bar{b}\rangle$ est donc identiquement nulle, et ceci quelle que soit la vitesse de balayage.

3.3 CRoISEMENTS DE DeUXIÈme ESPÈCE. - Par opposition au cas précédent, nous dirons que deux niveaux $\mid \bar{a}\left(\omega_{0}\right)>$ et $\mid \bar{b}\left(\omega_{0}\right)>$ qui se croisent en $\omega_{0}=\omega_{0 \mathrm{c}}$ forment un croisement de deuxième espèce si la probabilité de transition de $|\bar{a}\rangle$ vers $|\bar{b}\rangle$ est différente de 0 lorsqu'on balaie suffisamment rapidement le point de croisement.

Une telle situation est notamment réalisée lorsque $<\overline{\mathrm{a}}\left(\omega_{0 \mathrm{i}}\right)\left|\overline{\mathrm{b}}\left(\omega_{0 \mathrm{f}}\right)\right\rangle$ est différent de zéro. Supposons en effet que l'on fasse varier brusquement le paramètre $\omega_{0}$ de la valeur $\omega_{0 \mathrm{i}}$ à la valeur $\omega_{0 \mathrm{f}}$ (traversée très rapide du croisement de niveaux); le vecteur d'état du système n'a pas le temps d'évoluer et reste $\mid \bar{a}\left(\omega_{0 \mathrm{i}}\right)>$. La probabilité de transition de l'état $\left|\bar{a}\left(\omega_{0 \mathrm{i}}\right)\right\rangle$ vers l'état $\mid \bar{b}\left(\omega_{0 f}\right)>$ est dans le cadre de cette approximation soudaine égale à

$$
\left|<\overline{\mathrm{a}}\left(\omega_{0 \mathrm{i}}\right)\right| \overline{\mathrm{b}}\left(\omega_{0 \mathrm{f}}\right)>\left.\right|^{2}
$$

L'approximation soudaine représente toutefois un cas extrême et nous allons nous intéresser à la probabilité de transition $|\bar{a}>\rightarrow| \bar{b}>$ lorsque la vitesse de balayage du paramètre $\omega_{0}$ n'est pas infinie. Cette étude nous permettra, en outre, d'établir une classification des croisements de deuxième espèce.

Soient $|\bar{a}\rangle,|\bar{b}\rangle,|\bar{j}\rangle, \ldots$ les états propres de $\mathfrak{H}$ associés aux valeurs propres $E_{\mathrm{a}}, E_{\mathrm{b}}, E_{\mathrm{j}}, \ldots$ En général, les états propres et les valeurs propres de $H^{\mathcal{H}}$ sont fonctions de $\omega_{0}$. La variation de $\omega_{0}$ au cours du temps est décrite par la fonction $\omega_{0}(t): \omega_{0}(t)$ est égal à $\omega_{0 \mathrm{i}}$ pour $t=0$ et à $\omega_{0 \mathrm{f}}$ pour $t=T$. Dans ces conditions, le hamiltonien $\mathfrak{H}$ dépend explicitement du temps.
Cherchons une solution de l'équation de Schrödinger de la forme :

$$
\left|\psi(t)>=\sum_{\mathbf{j}} C_{\mathrm{j}}\left[\exp -1 \int_{0}^{t} E_{\mathrm{j}} \mathrm{d} \tau\right]\right| \overline{\mathrm{j}}>.
$$

En portant cette expression du vecteur d'état dans l'équation de Schrödinger, on obtient le système d'équations différentielles suivant pour les $C_{\mathrm{k}}\left(C_{\mathrm{k}}\right.$ représente, au facteur de phase exp $-\mathrm{i} \int_{0}^{t} E_{\mathrm{k}} \mathrm{d} \tau$ près, l'amplitude de probabilité de trouver le système dans l'état $\mid \overline{\mathrm{k}}>)$ :

$\frac{\mathrm{d} C_{\mathbf{k}}}{\mathrm{d} t}=-\sum_{\mathbf{j}} C_{\mathrm{j}}\left[\operatorname{exp~i} \int_{0}^{t}\left(E_{\mathrm{k}}-E_{\mathrm{j}}\right) \mathrm{d} \tau\right]\left\langle\overline{\mathrm{k}} \mid \frac{\mathrm{d} \overline{\mathrm{j}}}{\mathrm{d} t}\right\rangle$.

Dans l'évolution de $C_{\mathrm{b}}$, on peut distinguer deux termes :

$$
\begin{gathered}
\frac{\mathrm{d} C_{\mathrm{b}}}{\mathrm{d} t}=-C_{\mathrm{a}}\left[\operatorname{exp~i} \int_{0}^{t}\left(E_{\mathrm{b}}-E_{\mathrm{a}}\right) \mathrm{d} \tau\right] \times \\
\times\left\langle\overline{\mathrm{b}} \mid \frac{\mathrm{d} \overline{\mathrm{a}}}{\mathrm{d} \omega_{0}}\right\rangle \frac{\mathrm{d} \omega_{0}}{\mathrm{~d} i}-\sum_{\mathrm{j} \neq \mathrm{a}} C_{\mathrm{j}}\left[\operatorname{exp~i} \int_{0}^{t}\left(E_{\mathrm{b}}-E_{\mathrm{j}}\right) \mathrm{d} \tau\right] \\
\times\left\langle\overline{\mathrm{b}} \mid \frac{\mathrm{d} \overline{\mathrm{j}}}{\mathrm{d} \omega_{0}}\right\rangle \frac{\mathrm{d} \omega_{0}}{\mathrm{~d} t}
\end{gathered}
$$

Le premier terme de (3.3) correspond à une transition directe $|\bar{a}\rangle \rightarrow|\bar{b}\rangle$.

Les termes suivants correspondent à d'éventuelles transitions indirectes $|\bar{a}\rangle \rightarrow|\bar{j}>\rightarrow| \bar{b}\rangle$. Si la transition $|\bar{a}\rangle \rightarrow \mid \bar{b}>$ est possible (c'est-à-dire si $\left.\left\langle\bar{a} \mid \frac{d \bar{b}}{d \omega_{0}}\right\rangle \neq 0\right)$ nous dirons que l'ordre relatif des niveaux $|\bar{a}\rangle$ et $\mid \bar{b}>$ est égal à 0 ; si deux niveaux d'ordre relatif 0 se croisent, nous dirons qu'ils forment un croisement de deuxième espèce d'ordre 0 .

Si la transition $|\bar{a}\rangle \rightarrow|\bar{b}\rangle$ n'est pas possible (c'est-à-dire si $\left\langle\overline{\mathrm{a}} \mid \frac{\mathrm{d} \overline{\mathrm{b}}}{\mathrm{d} \omega_{0}}\right\rangle$ est identiquement nul) mais s'il existe une transition indirecte

$$
|\bar{a}\rangle \rightarrow|\bar{j}\rangle \rightarrow|\bar{b}\rangle
$$

via un seul état intermédiaire, nous dirons que l'ordre relatif des niveaux $\mid \bar{a}>$ et $\mid \bar{b}>$ est égal à 1 ; si deux niveaux d'ordre relatif égal à 1 se croisent, nous dirons qu'ils forment un croisement de deuxième espèce d'ordre 1. L'existence d'une transition via l'état intermédiaire $|\bar{j}\rangle$ s'exprime mathématiquement par la condition :

$$
\left\langle\bar{a} \mid \frac{d \bar{j}}{d \omega_{0}}\right\rangle\left\langle\bar{j} \mid \frac{d \bar{b}}{d \omega_{0}}\right\rangle \neq 0 .
$$

Nous verrons au paragraphe 3.5 que le croisement de deuxième espèce étudié dans la partie 2 est de ce type. 
De façon plus générale, s'il n'y a pas de transition directe entre les états $|\bar{a}\rangle$ et $\mid \bar{b}>$ et si la première transition indirecte fait intervenir une chaîne de $p$ états intermédiaires $\left|\bar{k}_{1}\right\rangle, \ldots,\left|\bar{k}_{p}\right\rangle$, nous dirons que l'ordre relatif des niveaux $|\overline{\mathrm{a}}\rangle$ et $|\overline{\mathrm{b}}\rangle$ est égal à $p\left({ }^{2}\right)$; si deux niveaux d'ordre relatif égal à $p$ se croisent, ils forment un croisement de deuxième espèce $d$ 'ordre $p$. On exprime mathématiquement que deux niveaux sont d'ordre relatif $p(p \geqslant 1)$ à l'aide des conditions suivantes :

$$
\left\langle\overline{\mathrm{a}} \mid \frac{\mathrm{d} \overline{\mathrm{b}}}{\mathrm{d} \omega_{0}}\right\rangle=0,
$$

(ii) pour toute chaîne de $q$ états intermédiaires $(q<p)$ :

$$
\left\langle\overline{\mathrm{a}} \mid \frac{\mathrm{d}_{1}}{\mathrm{~d} \omega_{0}}\right\rangle\left\langle\overline{\mathrm{k}}_{1} \mid \frac{\mathrm{d}_{2}}{\mathrm{~d} \omega_{0}}\right\rangle \ldots\left\langle\overline{\mathrm{k}}_{q} \mid \frac{\mathrm{d} \overline{\mathrm{b}}}{\mathrm{d} \omega_{0}}\right\rangle=0,
$$

(iii) il existe une chaîne de $p$ états intermédiaires telle que :

$$
\left\langle\overline{\mathrm{a}} \mid \frac{\mathrm{d} \overline{\mathrm{k}}_{1}}{\mathrm{~d} \omega_{0}}\right\rangle\left\langle\overline{\mathrm{k}}_{1} \mid \frac{\mathrm{d} \overline{\mathrm{k}}_{2}}{\mathrm{~d} \omega_{0}}\right\rangle \ldots\left\langle\overline{\mathrm{k}}_{p} \mid \frac{\mathrm{d} \overline{\mathrm{b}}}{\mathrm{d} \omega_{0}}\right\rangle \neq 0 .
$$

S'il n'y a aucune transition possible (directe ou indirecte) entre les états $|\bar{a}\rangle$ et $|\bar{b}\rangle$, nous dirons que l'ordre relatif de ces niveaux est infini. On montre dans l'appendice A que si deux niveaux sont d'ordre relatif infini, on peut trouver un opérateur de symétrie (c'est-à-dire un opérateur indépendant de $\omega_{0}$ et commutant avec $\mathcal{H}$ ) tel que $|\overline{\mathrm{a}}\rangle$ et $|\overline{\mathrm{b}}\rangle$ soient états propres de cet opérateur associés à des valeurs propres distinctes. Ceci prouve qu'un croisement entre deux niveaux d'ordre relatif infini est un croisement de première espèce.

L'existence d'une probabilité de transition entre deux niveaux qui se croisent pose immédiatement le problème de la validité du théorème adiabatique pour les croisements de niveaux de deuxième espèce. En fait, la démonstration du théorème adiabatique donnée par Kato [4] inclut tous les croisements de niveaux ; nous ne reprendrons pas ici cette démonstration, nous désirons seulement montrer physiquement comment les croisements de divers ordres se comportent vis-à-vis de ce théorème.

3.4 TraverséE ADIABATIQUE D'UN CROISEMENT. $\mathrm{La}$ démonstration habituelle du théorème adiabatique [5] se fait dans le cadre des hypothèses suivantes :

(i) Tous les niveaux sont discrets.

(ii) Il n'y a pas de croisement de niveaux dans le diagramme d'énergie.

On démontre alors que si le système se trouve à l'instant 0 pour la valeur $\omega_{0 \mathrm{i}}$ du paramètre dans l'état $\mid \overline{\mathrm{k}}>$ (cf. Fig. 6) et si l'on balaie le paramètre de la

(2) On peut définir l'ordre relatif de deux niveaux, indépendamment du fait qu'ils se croisent ou ne se croisent pas. valeur $\omega_{0 \mathrm{i}}$ à la valeur $\omega_{\text {of }}$ durant un temps $T$, le système se trouve à la fin du balayage dans une superposition linéaire des états $|\overline{\mathrm{k}}\rangle,|\overline{\mathrm{l}}>,| \overline{\mathrm{m}}\rangle, \ldots$ Cependant, à la limite où $T$ tend vers l'infini, les coefficients des états différents de $|\overline{\mathrm{k}}\rangle$ dans le développement du vecteur d'état tendent vers 0 comme $1 / T$. En d'autres termes, si l'on balaie suffisamment lentement le paramètre $\omega_{0}$, le système reste dans l'état se déduisant de l'état initial par continuité.

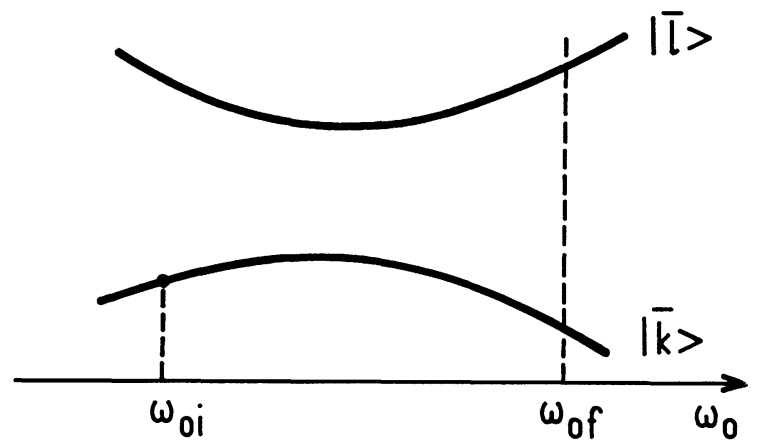

Fig. 6. - Lorsqu'on balaie $\omega_{0}$ de $\omega_{01}$ à $\omega_{0 \text { f }}$, la vitesse de rotation des axes $\left|\left\langle\overline{\mathrm{k}} \mid \frac{\mathrm{d} \overline{\mathrm{l}}}{\mathrm{d} t}\right\rangle\right|$ doit être petite devant la fréquence de Bohr $\left(E_{1}-E_{\mathrm{k}}\right) / h$ pour que le théorème adiabatique soit applicable.

On peut montrer que le théorème adiabatique est applicable si la vitesse de rotation des axes

$$
\left|\left\langle\overline{\mathrm{k}} \mid \begin{array}{c}
\mathrm{d} \overline{\mathrm{l}} \\
\overline{\mathrm{d} t}
\end{array}\right\rangle\right|
$$

est petite devant une fréquence qui est de l'ordre de grandeur de la fréquence de Bohr $\Omega_{\mathrm{k} 1}=E_{1}-E_{\mathrm{k}}$. L'existence de cette condition pourrait laisser planer un doute sur l'extension du théorème à un croisement de niveaux; en fait, il ne s'agit que d'une condition suffisante et on peut étendre le théorème adiabatique au cas où le niveau considéré traverse un certain nombre de niveaux discrets [4]. Dans le cas où le croisement est d'un ordre supérieur ou égal à 1 , ce résultat se comprend très aisément. Raisonnons d'abord sur le cas d'un croisement de première espèce : lorsque l'on balaie $\omega_{0}$ de $\omega_{0 \mathrm{i}}$ à $\omega_{0 \mathrm{f}}$ il n'y a pas de transitions possibles entre les états $|\bar{a}\rangle$ et $|\bar{b}\rangle$ qui se croisent ; donc du point de vue du théorème adiabatique tout se passe comme si le niveau $\mid \bar{b}>$ n'existait pas. En particulier, le théorème adiabatique sera certainement applicable si les $\left|\left\langle\overline{\mathrm{a}} \mid \frac{\mathrm{d} \overline{\mathrm{k}}}{\mathrm{d} t}\right\rangle\right|$ sont petits devant les fréquences de Bohr $\Omega_{\mathbf{k a}}(\mid \overrightarrow{\mathrm{k}}>$ étant un état différent de $\bar{b}>$, cf. Fig. 7).

Etudions maintenant le cas d'un croisement d'ordre 1 (si l'ordre du croisement est supérieur à 1 , les considérations suivantes s'étendent facilement). Supposons que les transitions entre $|\bar{a}\rangle$ et $|\bar{b}\rangle$ se font via un certain nombre de niveaux dont le niveau $\mid \overline{\mathrm{k}}>$. Pour 


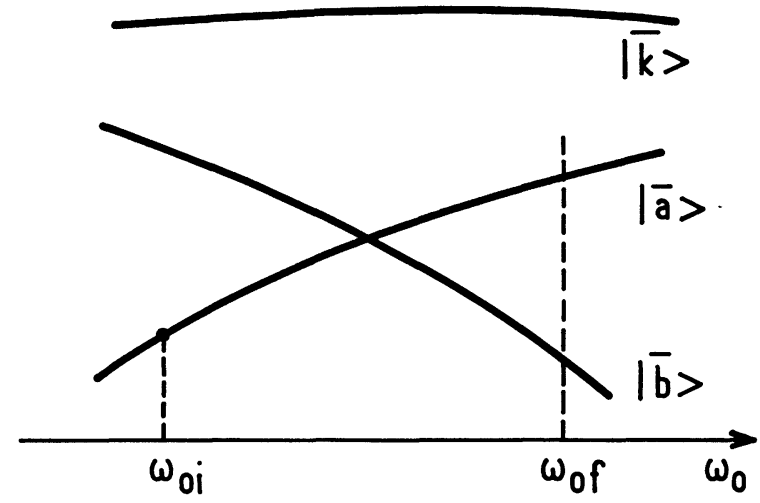

FIG. 7. - Pour un croisement de première espèce, la fréquence de $\operatorname{Bohr}\left(E_{\mathrm{a}}-E_{\mathrm{b}}\right) / h$ n'intervient pas dans la démonstration du théorème adiabatique et le fait que cette fréquence s'annule au point de croisement ne limite pas la portée de ce théorème. Il en est de même pour un croisement de deuxième espèce d'ordre supérieur ou égal à 1 .

qu'à l'instant $t=T$, la fonction d'onde du système soit fortement contaminée par le niveau $|\bar{b}\rangle$, il faut qu'elle soit encore plus fortement contaminée par l'état $\mid \overline{\mathrm{k}}>$ puisqu'on ne peut passer directement de $|\overline{\mathrm{a}}>\mathrm{a}| \overline{\mathrm{b}}>$. On voit donc que les conditions d'application du théorème adiabatique seront limitées non pas par l'existence du croisement, mais par la proximité du niveau $|\overline{\mathrm{k}}\rangle$.

L'extension du théorème adiabatique aux croisements d'ordre 0 est plus délicate ; elle nécessite l'emploi de la démonstration générale de Kato [4]. Les conditions de validité du théorème adiabatique en ces points de croisements doivent faire l'objet d'une étude spéciale. Ce problème est abordé dans la référence [6].

3. 5 EXEMPLE DE CROISEMENT DE DEUXIÈME ESPÈCE D'ORDRE 1. - Reprenons l'exemple simple étudié dans la partie 2 et montrons qu'il s'agit d'un croisement de deuxième espèce. Calculons pour cela le produit scalaire $\left\langle\overline{\mathrm{a}}\left(\omega_{01}\right) \mid \overline{\mathrm{b}}\left(\omega_{02}\right)\right\rangle$. Le développement de Rayleigh-Schrödinger à l'ordre 2 en $v / \omega$ des états $\mid \bar{a}>$ et $\mid \bar{b}>$ s'écrit :

$$
\begin{gathered}
\left|\overline{\mathrm{a}}\left(\omega_{01}\right)>=\right| \mathrm{a}>+\frac{v}{\omega_{01}-\omega} \mid \mathrm{i}>+ \\
+\frac{v}{\omega_{01}+\omega}\left|\mathrm{j}>+\frac{v^{2}}{\left(\omega_{01}^{2}-\omega^{2}\right)}\right| \mathrm{b}>+\cdots \\
\left|\overline{\mathrm{b}}\left(\omega_{02}\right)>=\right| \mathrm{b}>-\frac{v}{\omega_{02}+\omega} \mid \mathrm{i}>+ \\
+\frac{v}{-\omega_{02}+\omega}\left|\mathrm{j}>+\frac{v^{2}}{\omega_{02}^{2}-\omega^{2}}\right| \mathrm{a}>+\cdots
\end{gathered}
$$

Un calcul algébrique élémentaire donne alors :

$<\overline{\mathrm{a}}\left(\omega_{01}\right) \mid \overline{\mathrm{b}}\left(\omega_{02}\right)>=\frac{v^{2}\left(\omega_{01}-\omega_{02}\right)^{2}}{\left(\omega_{01}^{2}-\omega^{2}\right)\left(\omega_{02}^{2}-\omega^{2}\right)}+\cdots$
Le produit scalaire $<\overline{\mathrm{a}}\left(\omega_{01}\right) \mid \overline{\mathrm{b}}\left(\omega_{02}\right)>$ n'est donc nul que pour $\omega_{01}=\omega_{02}$ ce qui prouve bien, d'après les résultats du paragraphe 3.3 , que le croisement est de deuxième espèce.

On déduit également de l'expression (3.6) que :

$$
\left\langle\overline{\mathrm{a}}\left(\omega_{0}\right) \mid \frac{\mathrm{d} \overline{\mathrm{b}}}{\mathrm{d} \omega_{0}}\left(\omega_{0}\right)\right\rangle=0,
$$

ce qui montre que le croisement n'est pas d'ordre zéro. Par contre, en utilisant le développement de $|\mathrm{i}\rangle$, on obtient aisément :

$$
\left\langle\overline{\mathbf{a}}\left(\omega_{0}\right) \mid \frac{\mathrm{d}\left(\omega_{0}\right)}{\mathrm{d} \omega_{0}}\right\rangle\left\langle\overline{\mathrm{i}}\left(\omega_{0}\right) \mid \frac{\mathrm{d} \overline{\mathrm{b}}\left(\omega_{0}\right)}{\mathrm{d} \omega_{0}}\right\rangle \neq 0 .
$$

Le croisement est donc d'ordre $1\left({ }^{3}\right)$.

3.6 EXEMPLE DE CROISEMENT DE DEUXIÈME ESPÈCE D'ORDRE 0. - Considérons un spin $\frac{1}{2}$ placé dans un champ magnétique dont on fait varier simultanément la direction et l'amplitude. Supposons par exemple que le champ $\mathbf{H}_{0}$ est dans le plan $\mathrm{xOy}$ et appelons $\theta$ l'angle qu'il fait avec Ox (Fig. 8).

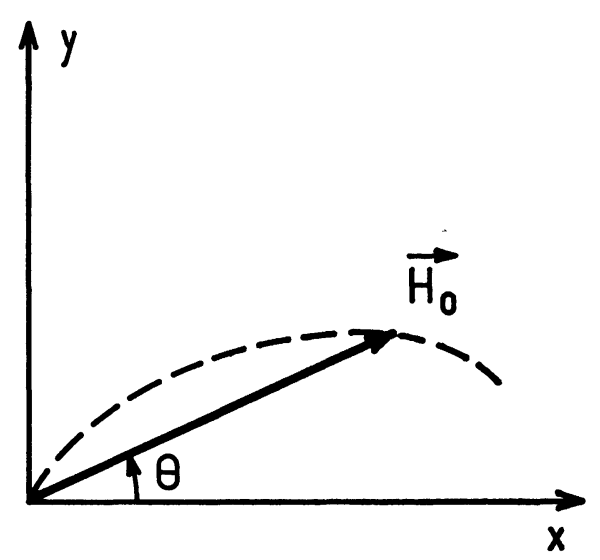

FIG. 8. - Champ magnétique $\mathbf{H}_{\mathbf{0}}$ dont on fait varier simultanément la direction et l'amplitude dans le plan xOy. Les niveaux d'énergie d'un spin $\frac{1}{2}$ plongé dans un tel champ forment en $\mathbf{H}_{0}=\mathbf{0}$ un croisement de deuxième espèce d'ordre 0 .

On suppose que $\theta$ est une certaine fonction de $\left|\mathbf{H}_{0}\right|$. Soit $\gamma_{\alpha}$ le rapport gyromagnétique du niveau considéré, le hamiltonien $\mathfrak{H e}$ s'écrit

$$
\mathscr{H}=\omega_{0} J_{\theta}
$$

où $\omega_{0}$ est égal à $-\gamma_{\alpha} H_{0}$.

Les états propres de $\mathscr{H}$ sont les états $\mid+>$ et $\mid->$ dans la direction $\theta$ que nous appellerons $\mid+(\theta)>$ et

(3) Ce résultat démontre à l'ordre 2 en $v / \omega$ est en fait vrai à tous les ordres comme le montre un calcul direct fait en appendice $B$. 
$\mid-(\theta)>$; ils sont reliés aux états $\mid+>$ et $\mid->$ par rapport à $\mathrm{Oz}$ par les relations suivantes :

$\mid+(\theta)>=$

$=\frac{1}{\sqrt{2}} \exp \left(-\mathrm{i} \frac{\theta}{2}\right)\left|+>+\frac{1}{\sqrt{2}} \exp \left(\mathrm{i} \frac{\theta}{2}\right)\right|->$

$$
\begin{aligned}
\mid-(\theta) & >= \\
& =\frac{1}{\sqrt{2}} \exp \left(-\mathrm{i} \frac{\theta}{2}\right)\left|+>-\frac{1}{\sqrt{2}} \exp \left(\mathrm{i} \frac{\theta}{2}\right)\right|->.
\end{aligned}
$$

Les valeurs propres de $\mathscr{H}$ associées à $\mid+(\theta)>$ et $|-(\theta)\rangle$ sont respectivement $\omega_{0} / 2$ et $-\omega_{0} / 2$.

Les niveaux d'énergie $\mid+(\theta)>$ et $\mid-(\theta)>$ se croisent quand le champ magnétique $\mathbf{H}_{0}$ passe par la valeur zéro. Pour préciser l'ordre de ce croisement, nous allons calculer

$$
\begin{gathered}
\left\langle-(\theta) \mid \frac{\mathrm{d}}{\mathrm{d} \omega_{0}}+(\theta)\right\rangle \\
\left|\frac{\mathrm{d}-}{\mathrm{d} \omega_{0}}+(\theta)\right\rangle=-\frac{1}{2 \sqrt{2}} \frac{\mathrm{d} \theta}{\mathrm{d} \omega_{0}} \times \\
\times\left[\exp \left(-\mathrm{i} \frac{\theta}{2}\right)\left|+>-\exp \left(\mathrm{i} \frac{\theta}{2}\right)\right|->\right]
\end{gathered}
$$

d'où l'on déduit :

$$
\left\langle-(\theta) \mid \frac{\mathrm{d}}{\mathrm{d} \omega_{0}}+(\theta)\right\rangle=-\frac{1}{2} \frac{\mathrm{d} \theta}{\mathrm{d} \omega_{0}} .
$$

On en déduit que, si le champ magnétique $\boldsymbol{H}_{0}$ varie simultanément en amplitude et direction $\left(\mathrm{d} \theta / \mathrm{d} \omega_{0} \neq 0\right)$, alors le croisement de niveaux est un croisement d'ordre 0 .

Nous avons ainsi donné aux paragraphes (3.5) et (3.6) des exemples de croisements d'ordre 1 et 0 . Nous verrons, dans l'article suivant, que l'on peut trouver dans le diagramme d'énergie d'un atome habillé par des photons de radiofréquence un grand nombre d'exemples de croisements de niveaux d'ordre $0,1,2, \ldots, n, \ldots$

\section{Lien avec le théorème de Von Neumann et Wigner.} - Dans la partie précédente, nous avons montré que les croisements de niveaux de deuxième espèce pouvaient être définis directement à partir du hamiltonien total $\mathcal{H}$ : ce sont des croisements entre des niveaux qui ne sont pas de symétries différentes. L'existence de tels croisements semble cependant incompatible avec le théorème de Von Neumann et Wigner; l'énoncé qu'on en donne généralement [2] stipule que seuls peuvent se croiser des niveaux de symétries différentes. Pour éclaircir ce point, nous allons reprendre la démonstration $\mathrm{du}$ théorème de Von Neumann et Wigner et montrer qu'en fait elle n'exclut pas les croisements de deuxième espèce.
4.1 LA DÉMONSTRATION USUELLE DU THÉORÈME DE Von NeumanN ET WigneR. - Dans la référence [2], ce théorème est démontré de la façon suivante : on considère deux niveaux $|\overline{\mathrm{a}}\rangle$ et $\mid \overline{\mathrm{b}}>$ dont les énergies $E_{\mathrm{a}}$ et $E_{\mathrm{b}}$ sont très voisines pour une certaine valeur du paramètre $\omega_{0}$ et on étudie s'il est possible d'égaliser $E_{\mathrm{a}}$ et $E_{\mathrm{b}}$ en faisant varier $\omega_{0}$ de $\delta \omega_{0}$ (cf. Fig. 9).

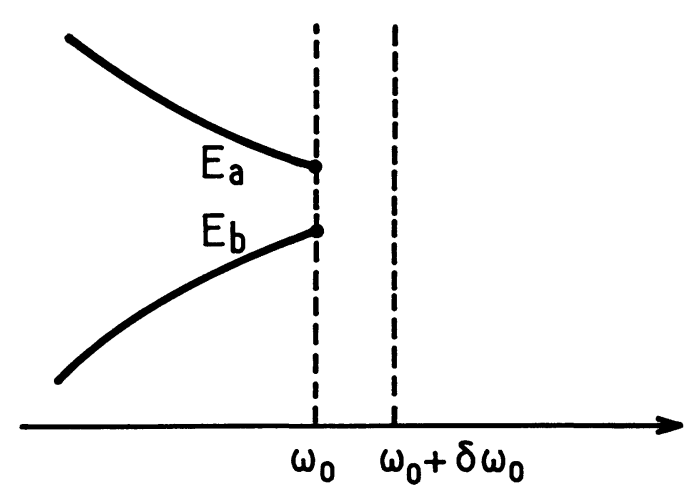

FIG. 9. - Deux niveaux a et $\mathrm{b}$ d'un hamiltonien $\mathscr{H}\left(\omega_{0}\right)$ ont en $\omega_{0}$ des énergies très voisines. On étudie s'il est possible d'égaliser ces énergies en faisant varier $\omega_{0}$ de $\delta \omega_{0}$.

Le hamiltonien en $\omega_{0}$ est $\mathcal{H}\left(\omega_{0}\right)$; en $\left(\omega_{0}+\delta \omega_{0}\right)$ il vaut

$$
\mathscr{H e}\left(\omega_{0}\right)+\frac{\mathrm{d} \mathscr{H}}{\mathrm{d} \omega_{0}} \delta \omega_{0}
$$

Posons

$$
W=\frac{\mathrm{d} \mathscr{H}}{\mathrm{d} \omega_{0}} \delta \omega_{0}
$$

Pour trouver les valeurs propres de $\mathcal{H}+W$, on traite $W$ comme une perturbation devant $\mathscr{H}$; cependant, ${ }_{i}^{*} W$ pouvant être du même ordre de grandeur que $E_{\mathrm{a}}{ }^{\prime}-E_{\mathrm{b}}$, il faut diagonaliser $\mathcal{H}+W$ dans le sous-espace engendré par $\mid \bar{a}\left(\omega_{0}\right)>$ et $\mid \bar{b}\left(\omega_{0}\right)>$. Il faut donc résoudre l'équation :

$$
\text { Dét }\left|\begin{array}{ll}
E_{\mathrm{a}}+W_{\mathrm{aa}}-E & W_{\mathrm{ab}} \\
W_{\mathrm{ba}} & E_{\mathrm{b}}+W_{\mathrm{bb}}-E
\end{array}\right|=0
$$

dont les solutions sont $E_{+}$et $E_{-}$:

$$
\begin{aligned}
E_{ \pm}=\frac{1}{2} & \left(E_{\mathrm{a}}+E_{\mathrm{b}}+W_{\mathrm{aa}}+W_{\mathrm{bb}}\right) \pm \\
& \pm \sqrt{\frac{1}{4}\left(E_{\mathrm{a}}-E_{\mathrm{b}}+W_{\mathrm{aa}}-W_{\mathrm{bb}}\right)^{2}+\left|W_{\mathrm{ab}}\right|^{2}} .
\end{aligned}
$$

Les niveaux ne peuvent se croiser que si $E_{+}$est égal à

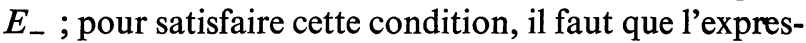
sion sous le radical soit nulle ; il faut par suite réaliser simultanément :

$$
\begin{aligned}
E_{\mathrm{a}}-E_{\mathrm{b}}+W_{\mathrm{aa}}-W_{\mathrm{bb}} & =0 \\
W_{\mathrm{ab}} & =0 .
\end{aligned}
$$


On obtient ainsi au moins deux équations $\left({ }^{4}\right)$ pour la seule inconnue $\delta \omega_{0}$. Il sera donc généralement impossible de satisfaire simultanément les deux conditions (4.3) et (4.4). Une condition suffisante pour qu'il y ait croisement est que $W_{\mathrm{ab}}$ soit identiquement nul pour toute valeur de $\omega_{0}$. La condition (4.4) disparaît alors et il n'y a plus qu'une seule équation (4.3) à une inconnue, qui donne en général une solution pour $\delta \omega_{0}$.

Si $|\bar{a}\rangle$ et $|\bar{b}\rangle$ sont de symétries différentes $W_{\text {ab }}$ est nul ; en effet, il existe alors un opérateur $L$ indépendant de $\omega_{0}$ et commutant avec $\mathscr{H}$

$$
[L, H]=0 \text {. }
$$

On peut dériver cette expression par rapport à $\omega_{0}$ :

$$
\left[L, \frac{\mathrm{d} \mathscr{H}}{\mathrm{d} \omega_{0}}\right]=0 \text {. }
$$

Un élément de matrice de $W=\frac{\mathrm{d} \mathscr{H}}{\mathrm{d} \omega_{0}} \delta \omega_{0}$ pris entre deux vecteurs propres de $L$ correspondant à des valeurs propres distinctes est donc nul.

4.2 ANALYSE DE LA DÉMONSTRATION. - La démonstration précédente fait intervenir deux conditions suffisantes successives; d'abord, on écrit qu'il suffit que $W_{\mathrm{ab}}$ soit identiquement nul pour que les niveaux se croisent; ensuite on écrit que, pour que $W_{\mathrm{ab}}$ soit nul, il suffit que les niveaux soient de symétries différentes. Nous allons montrer qu'en fait on peut donner une expression exacte de $W_{\text {ab }}$ qui permet de s'affranchir de la seconde condition suffisante. En effet

$$
W_{\mathrm{ab}}=\left\langle\overline{\mathrm{a}}\left|\frac{\mathrm{d} \mathscr{H}}{\mathrm{d} \omega_{0}}\right| \overline{\mathrm{b}}\right\rangle \delta \omega_{0}
$$

et :

$$
\begin{aligned}
\left\langle\overline{\mathrm{a}}\left|\frac{\mathrm{d} \mathscr{H}}{\mathrm{d} \omega_{0}}\right| \overline{\mathrm{b}}\right\rangle=\frac{\mathrm{d}}{\mathrm{d} \omega_{0}}\langle\overline{\mathrm{a}}|\mathcal{H}| \overline{\mathrm{b}}\rangle- \\
-\left\langle\frac{\mathrm{d} \overline{\mathrm{a}}}{\mathrm{d} \omega_{0}}|\mathcal{H}| \overline{\mathrm{b}}\right\rangle-\left\langle\overline{\mathrm{a}}|\mathcal{H}| \frac{\mathrm{d} \overline{\mathrm{b}}}{\mathrm{d} \omega_{0}}\right\rangle
\end{aligned}
$$

$|\overline{\mathrm{a}}\rangle$ et $|\overline{\mathrm{b}}\rangle$ étant états propres de $\mathcal{H}$ associés aux valeurs propres $E_{\mathrm{a}}$ et $E_{\mathrm{b}}$, il vient :

$\left\langle\overline{\mathrm{a}}\left|\frac{\mathrm{d} \mathscr{H}}{\mathrm{d} \omega_{0}}\right| \overline{\mathrm{b}}\right\rangle=-E_{\mathrm{b}}\left\langle\frac{\mathrm{d} \overline{\mathrm{a}}}{\mathrm{d} \omega_{0}} \mid \overline{\mathrm{b}}\right\rangle-E_{\mathrm{a}}\left\langle\overline{\mathrm{a}} \mid \frac{\mathrm{d} \overline{\mathrm{b}}}{\mathrm{d} \omega_{0}}\right\rangle$.

En remarquant que

$$
\left\langle\frac{\mathrm{d} \overline{\mathrm{a}}}{\mathrm{d} \omega_{0}} \mid \overline{\mathrm{b}}\right\rangle+\left\langle\overline{\mathrm{a}} \mid \frac{\mathrm{d} \overline{\mathrm{b}}}{\mathrm{d} \omega_{0}}\right\rangle=0
$$

on obtient finalement :

$$
W_{\mathrm{ab}}=\left(E_{\mathrm{b}}-E_{\mathrm{a}}\right)\left\langle\overline{\mathrm{a}} \mid \frac{\mathrm{d} \overline{\mathrm{b}}}{\mathrm{d} \omega_{0}}\right\rangle \delta \omega_{0} .
$$

(4) Il y a trois équations si $W_{\mathrm{ab}}$ est complexe.
Il s'ensuit que les conclusions habituelles du théorème de Von Neumann et Wigner s'étendent à tout couple de niveaux dont l'ordre relatif est supérieur ou égal à 1 . Ce résultat se comprend facilement: lorsque deux niveaux s'anticroisent, on peut se convaincre aisément à partir de l'expression des états propres au voisinage de l'anticroisement que $\left\langle\overline{\mathrm{a}} \mid \frac{\mathrm{d} \overline{\mathrm{b}}}{\mathrm{d} \omega_{0}}\right\rangle$ est différent de zéro ; deux niveaux ne peuvent s'anticroiser que si leur ordre relatif est 0 . Par suite, rien ne s'oppose à ce que deux niveaux d'ordre relatif 1 se croisent, même s'ils ont des symétries identiques.

On remarque par ailleurs que la démonstration précédente ne donne aucun renseignement en ce qui concerne les croisements d'ordre 0 : ces derniers ne peuvent pas, en effet, être inclus dans la démonstration si l'on se limite à la condition suffisante $W_{\mathrm{ab}} \equiv 0$, puisqu'ils correspondent justement à des cas où $W_{\mathrm{ab}}$ n'est pas identiquement nul. On pourra, cependant, prévoir l'existence de certains croisements d'ordre 0 s'il apparaît pour une valeur particulière $\omega_{0 p}$ du paramètre $\omega_{0}$ une symétrie ponctuelle entraînant dégénérescence; plusieurs niveaux viennent alors se croiser en $\omega_{0 p}$. L'ordre d'un tel croisement, qui a priori peut être quelconque, doit faire l'objet d'une étude séparée. Par exemple, le croisement d'ordre 0 que nous avons présenté au paragraphe 3.6 est lié à l'existence d'une telle symétrie : en effet, pour la valeur particulière 0 du champ magnétique, le système possède l'invariance de rotation et les niveaux doivent être dégénérés. Bien que l'ordre relatif des niveaux soit 0 , on voit sur cet exemple que l'existence d'une symétrie ponctuelle les empêche de s'anticroiser. Nous trouverons dans le diagramme d'énergie de l'atome habillé un autre exemple, moins simple, de croisement lié à une symétrie ponctuelle et nous montrerons que, suivant la polarisation de la radiofréquence, ce croisement peut être soit de première espèce, soit de deuxième espèce d'ordre 1 , soit de deuxième espèce d'ordre 0 .

Conclusion. - Nous avons pu ainsi à partir de deux approches distinctes, l'une perturbative, l'autre fondée directement sur les propriétés des vecteurs propres du hamiltonien total, caractériser deux types de croisements susceptibles d'apparaître sur un diagramme d'énergie.

Nous avons dégagé sur des exemples simples des phénomènes physiques permettant de différencier clairement ces deux situations (caractéristiques des résonances observables au voisinage de ces croisements, transition d'un niveau à l'autre lors d'un balayage suffisamment rapide). Nous avons enfin montré que le théorème de Wigner-Von Neumann, formulé de facon correcte, n'excluait pas l'existence des croisements de deuxième espèce.

Dans l'article suivant, nous montrons que les notions précédentes sont très utiles pour la classification et l'interprétation des résonances observables sur un atome habillé par des photons de radiofréquence. 
Remerciements. - Les auteurs tiennent à remercier M. S. Feneuille pour les fructueuses discussions qu'ils ont eues ensemble au sujet de cet article.

Appendice A : Ordre relatif infini et existence d'une symétrie. - Nous allons montrer que si deux niveaux $|\bar{a}\rangle$ et $|\bar{b}\rangle$ sont d'ordre relatif infini, on peut définir un opérateur de symétrie $L$ indépendant de $\omega_{0}$, commutant avec $\mathcal{H}$ et tel que $|\overline{\mathrm{a}}\rangle$ et $|\overline{\mathrm{b}}\rangle$ soient vecteurs propres de cet opérateur associés à des valeurs propres distinctes.

Pour cela, nous allons classer les états propres de $\mathscr{H}\left(\omega_{01}\right)$ en trois ensembles définis respectivement de la façon suivante :

- $\mathscr{F}_{\mathrm{a}}$ est composé de $|\overline{\mathrm{a}}\rangle$ et de tous les vecteurs propres de $\mathcal{H}\left(\omega_{01}\right)$ d'ordre relatif fini avec $|\overline{\mathrm{a}}\rangle$.

- $\mathcal{F}_{\mathrm{b}}$ est composé de $|\overline{\mathrm{b}}\rangle$ et de tous les vecteurs propres de $\mathscr{H}\left(\omega_{01}\right)$ d'ordre relatif fini avec $|\bar{b}\rangle$.

- $\mathscr{F}_{\mathrm{c}}$ est composé de tous les vecteurs propres de $\mathcal{H}\left(\omega_{01}\right)$ d'ordre relatif infini avec $|\overline{\mathrm{a}}\rangle$ et $|\overline{\mathrm{b}}\rangle$.

Nous allons montrer que ces trois ensembles sont disjoints. Supposons par exemple, qu'il existe un état $\mid \overline{1}>$ commun à $\mathscr{F}_{\mathbf{a}}$ et à $\mathcal{F}_{\mathbf{b}}$. Puisque $\mid \overline{1}>$ appartient à $\mathcal{F}_{\mathrm{a}}$, on peut le relier à $|\overline{\mathrm{a}}\rangle$ par une chaîne finie ; puisque $\mid \overline{1}>$ appartient à $\mathscr{F}_{b}$ on peut le relier à $|\vec{b}\rangle$ par une chaîne finie ; en unissant ces deux chaînes, on trouve donc une chaîne finie reliant $|\bar{a}\rangle$ à $|\bar{b}\rangle$, ce qui est contraire à l'hypothèse.

Soient respectivement $\mathcal{G}_{\mathrm{a}}, \mathcal{G}_{\mathrm{b}}$ et $\mathcal{G}_{\mathrm{c}}$ les sous-espaces engendrés par $\mathscr{F}_{a}, \mathscr{F}_{b}$ et $\mathcal{F}_{c}$; puisque $\mathscr{F}_{a}, \mathscr{F}_{b}$ et $\mathscr{F}_{\mathrm{c}}$ sont disjoints et puisque $\left(\mathscr{F}_{\mathrm{a}} \cup \mathscr{F}_{\mathrm{b}} \cup \mathscr{F}_{\mathrm{c}}\right)$ est une base de l'espace, tout vecteur se décompose de façon unique suivant $\mathcal{G}_{\mathrm{a}}, \mathcal{G}_{\mathrm{b}}$ et $\mathcal{G}_{\mathrm{c}}$.

Soit $\mid \overrightarrow{\mathrm{k}}_{\mathrm{a}}>$ un élément de $\mathcal{F}_{\mathrm{a}}$; nous allons montrer que $\frac{\mathrm{d}}{\mathrm{d} \omega_{0}} \mid \mathrm{k}_{\mathrm{a}}>$ appartient à $\mathcal{S}_{\mathrm{a}}$. Supposons en effet que la projection de $\frac{\mathrm{d}}{\mathrm{d} \omega_{0}} \mid \overline{\mathrm{k}}_{\mathrm{a}}>$ sur un vecteur $\left|\overline{\mathrm{k}}_{\mathrm{b}}\right\rangle$ de $\mathcal{F}_{\mathrm{b}}$ soit non nulle :

$$
\left\langle\overline{\mathrm{k}}_{\mathrm{b}} \mid \frac{\mathrm{d}}{\mathrm{d} \omega_{0}} \overline{\mathrm{k}}_{\mathrm{a}}\right\rangle \neq 0 .
$$

Puisque $\mid \overline{\mathrm{k}}_{\mathrm{b}}>$ appartient à $\mathcal{F}_{\mathrm{b}}$, il est relié à $\mid \overline{\mathrm{b}}>$ par une chaîne finie; puisque $\left|\overline{\mathrm{k}}_{\mathrm{a}}\right\rangle$ appartient à $\mathcal{F}_{\mathrm{a}}$, il est relié à $|\bar{a}\rangle$ par une chaîne finie ; en rajoutant le maillon $\left\langle\overline{\mathrm{k}}_{\mathrm{b}} \mid \frac{\mathrm{d}}{\mathrm{d} \omega_{0}} \overline{\mathrm{k}}_{\mathrm{a}}\right\rangle$ on pourrait relier $|\overline{\mathrm{a}}\rangle$ à $|\bar{b}\rangle$ par une chaîne finie, ce qui est contraire à l'hypothèse. On démontre de façon analogue que la projection de $\frac{\mathrm{d} \mid \overline{\mathrm{k}}_{\mathrm{a}}>}{\mathrm{d} \omega_{0}} \operatorname{sur} \mathcal{G}_{\mathrm{c}}$ est nulle. On a donc:

$$
\frac{\mathrm{d} \mid \overline{\mathrm{k}}_{\mathrm{a}}>}{\mathrm{d} \omega_{0}}=\sum_{\mathrm{I} \overline{\mathrm{u}}_{\mathrm{i}}>\in \mathcal{F}_{\mathrm{a}}} \lambda_{\mathrm{i}} \mid \overline{\mathrm{u}}_{\mathrm{i}}>\text {. }
$$

En dérivant la relation (A.1) on obtient :

$$
\frac{\mathrm{d}^{2} \mid \overline{\mathrm{k}}_{\mathrm{a}}>}{\mathrm{d} \omega_{0}^{2}}=\sum_{\mathrm{I} \overline{\mathrm{u}}_{\mathrm{i}}>\in \mathcal{F}_{\mathrm{a}}} \frac{\mathrm{d} \lambda_{\mathrm{i}}}{\mathrm{d} \omega_{0}} \mid \overline{\mathrm{u}}_{\mathrm{i}}>+\lambda_{\mathrm{i}} \frac{\mathrm{d} \mid \overline{\mathrm{u}}_{\mathrm{i}}>}{\mathrm{d} \omega_{0}} \text {. }
$$

Mais comme on l'a démontré précédemment, la dérivée de tout vecteur de $\mathscr{F}_{\mathrm{a}}$ appartient à $\mathcal{G}_{\mathrm{a}}$; par suite, $\frac{\mathrm{d}\left|\overline{\mathrm{u}}_{\mathrm{i}}\right\rangle}{\mathrm{d} \omega_{0}}$ appartient à $\mathcal{G}_{\mathrm{a}}$, et d'après la relation (A.2) $\frac{\mathrm{d}^{2} \mid \overline{\mathrm{k}}_{\mathrm{a}}}{\mathrm{d} \omega_{0}^{2}} \geq$ appartient également à $\mathcal{G}_{\mathrm{a}}$. En faisant une démonstration par récurrence, on étend sans peine ce résultat à $\frac{\mathrm{d}^{n} \mid \overline{\mathrm{k}}_{\mathrm{a}}>}{\mathrm{d} \omega_{0}^{n}}$; si $\mid \overline{\mathrm{k}}_{\mathrm{a}}>$ appartient à $\mathcal{F}_{\mathrm{a}}$ toutes ses dérivées appartiennent à $\mathcal{G}_{\mathbf{a}}$.

Toutes les dérivées de $\left|\overline{\mathbf{k}}_{\mathrm{a}}\right\rangle$ appartenant à $\mathcal{G}_{\mathrm{a}}$, le vecteur $\mid \overrightarrow{\mathbf{k}}_{\mathbf{a}}\left(\omega_{02}\right)>$ dont le développement de Taylor s'écrit :

$$
\begin{aligned}
& \left|\overline{\mathrm{k}}_{\mathrm{a}}\left(\omega_{02}\right)>=\right| \overline{\mathrm{k}}_{\mathrm{a}}\left(\omega_{01}\right)>+\left(\omega_{02}-\omega_{01}\right) \times \\
& \quad \times\left|\frac{\mathrm{d} \overline{\mathrm{k}}_{\mathrm{a}}\left(\omega_{01}\right)}{\mathrm{d} \omega_{0}}\right\rangle+\cdots+\frac{\left(\omega_{02}-\omega_{01}\right)^{n}}{n !}\left|\frac{\mathrm{d}^{n} \overline{\mathrm{k}}_{\mathrm{a}}\left(\omega_{01}\right)}{\mathrm{d} \omega_{0}^{n}}\right\rangle+\cdots
\end{aligned}
$$

appartient également à $\mathcal{G}_{\mathrm{a}}$.

Ceci prouve que le sous-espace $\mathcal{S}_{\mathrm{a}}$ que nous avons défini pour une valeur particulière $\omega_{01}$ de $\omega_{0}$ est en fait indépendant de $\omega_{0}$ et que l'ensemble des états propres de $\mathcal{H}\left(\omega_{0}\right)$ d'ordre relatif fini avec $\left|\overrightarrow{\mathbf{a}}\left(\omega_{0}\right)\right\rangle$ forme une base de $\mathcal{G}_{a}$, et ceci quel que soit $\omega_{0}$. On en déduit que le projecteur sur $\mathcal{G}_{\mathrm{a}}$ (que nous appellerons $\left.P_{\mathrm{a}}\right)$ est un opérateur de symétrie : en effet, $P_{\mathrm{a}}$ et $\mathscr{H}\left(\omega_{0}\right)$ ont les mêmes vecteurs propres, donc $P_{\mathrm{a}}$ et $\mathcal{H}\left(\omega_{0}\right)$ commutent ; d'autre part, $P_{\mathrm{a}}$ est un opérateur indépendant de $\omega_{0}$ (projecteur sur le sous-espace $\mathcal{G}_{\mathrm{a}}$ indépendant de $\omega_{0}$ ). Enfin $|\overline{\mathrm{a}}\rangle$ et $|\overline{\mathrm{b}}\rangle$ sont états propres de $P_{\mathrm{a}}$ associés à des valeurs propres distinctes 1 et 0 .

On a ainsi montré que si deux niveaux sont d'ordre relatif infini, il existe un opérateur de symétrie dont ils sont états propres associés à des valeurs propres distinctes. Cet opérateur de symétrie n'est d'ailleurs pas unique puisque toute combinaison linéaire de $P_{\mathrm{a}}$, $P_{\mathrm{b}}$ et $P_{\mathrm{c}}\left(P_{\mathrm{b}}\right.$ et $P_{\mathrm{c}}$ étant les projecteurs sur $\mathcal{G}_{\mathrm{b}}$ et $\left.\mathcal{G}_{\mathrm{c}}\right)$ peut être considérée comme un opérateur de symétrie.

Appendice B : Ordre du croisement du modèle de la partie 2. - Nous allons montrer que la relation

$$
\left\langle\bar{a} \mid \frac{\mathrm{d} \bar{b}}{\mathrm{~d} \omega_{0}}\right\rangle=0
$$

est vraie à tous les ordres en $v / \omega$. Comme il est facile de constater que

$$
\left\langle\overline{\mathrm{a}}\left(\omega_{0}\right) \mid \frac{\mathrm{d} \overline{\mathrm{b}}\left(\omega_{0}\right)}{\mathrm{d} \omega_{0}}\right\rangle\left\langle\overline{\mathrm{i}}\left(\omega_{0}\right) \mid \frac{\mathrm{d} \overline{\mathrm{b}}\left(\omega_{0}\right)}{\mathrm{d} \omega_{0}}\right\rangle
$$

est différent de zéro, cela prouvera que le croisement du modèle étudié dans la partie 2 est d'ordre 1 .

On définit un opérateur $K$ antiunitaire par son action sur les états non perturbés $|\mathrm{a}\rangle,|\mathrm{b}\rangle,|\mathrm{i}\rangle$, $\mid \mathrm{j}>$. 


$$
\begin{aligned}
& K|\mathrm{a}>=-| \mathrm{b}> \\
& K|\mathrm{~b}>=-| \mathrm{a}> \\
& K|\mathrm{i}>=| \mathrm{j}> \\
& K|\mathrm{j}>=| \mathrm{i}>.
\end{aligned}
$$

On vérifie immédiatement que $K$ anticommute avec $\mathcal{H}_{0}$ et avec $V$. On en déduit que $K$ anticommute avec $\mathscr{H}=\mathfrak{H}_{0}+V$. Faisons agir l'anticommutateur $[K, \mathscr{H e}]_{+}$ sur $|\bar{a}\rangle$, on obtient :

$$
K \mathscr{H}\left|\overline{\mathrm{a}}>+H_{K}\right| \overline{\mathrm{a}}>=0
$$

d'où

$$
\mathscr{H} K\left|\overline{\mathrm{a}}>=-\omega_{0}^{\prime} K\right| \overline{\mathrm{a}}>\mathscr{}
$$

Cette dernière relation prouve que $K \mid \overline{\mathrm{a}}>$ est proportionnel à $|\bar{b}\rangle$

$$
K\left|\overline{\mathrm{a}}>=\rho \mathrm{e}^{\mathrm{i} \varphi}\right| \overline{\mathrm{b}}>.
$$

De la même façon, on démontre que $K \mid \bar{b}>$ est proportionnel à $|\bar{a}\rangle$

$$
K|\overline{\mathrm{b}}\rangle=\rho^{\prime} \mathrm{e}^{\mathrm{i} \varphi^{\prime}} \mid \overline{\mathrm{a}}>.
$$

De l'identité $K^{2}=1$, on déduit alors

$$
K^{2}|\overline{\mathrm{a}}\rangle=|\overline{\mathrm{a}}\rangle \text {. }
$$

Soit

$$
\rho \rho^{\prime} \mathrm{e}^{\mathrm{i}\left(\varphi^{\prime}-\varphi\right)}=1
$$

d'où

et

$$
\rho \rho^{\prime}=1
$$

$$
\varphi=\varphi^{\prime} .
$$

L'égalité $K=K^{+}$entraîne que

$$
<\overline{\mathrm{a}} \mid[K|\overline{\mathrm{b}}\rangle]=\langle\overline{\mathrm{a}}|\left[K^{+}|\overline{\mathrm{b}}\rangle\right],
$$

mais puisque $K$ est antiunitaire :

$$
<\overline{\mathrm{a}} \mid\left[K^{+}|\overline{\mathrm{b}}\rangle\right]=\langle\overline{\mathrm{b}}|[K|\overline{\mathrm{a}}\rangle]
$$

donc

$$
\langle\overline{\mathrm{a}}|[K|\overline{\mathrm{b}}\rangle]=\langle\overline{\mathrm{b}}|[K|\overline{\mathrm{a}}\rangle] .
$$

Soit

$$
\rho^{\prime} \mathrm{e}^{\mathrm{i} \varphi^{\prime}}=\rho \mathrm{e}^{\mathrm{i} \varphi} .
$$

En comparant (A.15) et (A.11), on obtient

$$
\rho=\rho^{\prime}=1 \text {. }
$$

Calculons enfin :

$$
\begin{gathered}
\left\langle\overline{\mathrm{a}} \mid \frac{\mathrm{d} \overline{\mathrm{b}}}{\mathrm{d} \omega_{0}}\right\rangle \\
\left\langle\overline{\mathrm{a}} \mid \frac{\mathrm{d} \overline{\mathrm{b}}}{\mathrm{d} \omega_{0}}\right\rangle=\langle\overline{\mathrm{a}}|\left[K^{+} K\left|\frac{\mathrm{d} \overline{\mathrm{b}}}{\mathrm{d} \omega_{0}}\right\rangle\right]
\end{gathered}
$$

d'où

$$
\left\langle\overline{\mathrm{a}} \mid \frac{\mathrm{d} \overline{\mathrm{b}}}{\mathrm{d} \omega_{0}}\right\rangle=\langle\overline{\mathrm{a}}|\left[K^{+} \frac{\mathrm{d}}{\mathrm{d} \omega_{0}} K|\overline{\mathrm{b}}\rangle\right] .
$$

C'est-à-dire :

$$
\left\langle\overline{\mathrm{a}} \mid \frac{\mathrm{d} \overline{\mathrm{b}}}{\mathrm{d} \omega_{0}}\right\rangle=\langle\overline{\mathrm{a}}|\left[K^{+} \frac{\mathrm{d}}{\mathrm{d} \omega_{0}} \mathrm{e}^{\mathrm{i} \varphi}|\overline{\mathrm{a}}\rangle\right]
$$

et

$$
\begin{aligned}
\left\langle\overline{\mathrm{a}} \mid \frac{\mathrm{d} \bar{b}}{\mathrm{~d} \omega_{0}}\right\rangle & =\mathrm{e}^{-\mathrm{i} \varphi}\langle\overline{\mathrm{a}}| K^{+}\left[\frac{\mathrm{d}}{\mathrm{d} \omega_{0}}|\overline{\mathrm{a}}\rangle\right]- \\
& -\mathrm{i} \frac{\mathrm{d} \varphi}{\mathrm{d} \omega_{0}} \mathrm{e}^{-\mathrm{i} \varphi}\langle\overline{\mathrm{a}}|\left[K^{+}|\overline{\mathrm{a}}\rangle\right] .
\end{aligned}
$$

Le dernier terme de (A.20) est nul puisque $K^{+}|\overline{\mathrm{a}}\rangle$ est proportionnel à $\mid \bar{b}>$. On obtient :

$$
\left\langle\bar{a} \mid \frac{d \bar{b}}{d \omega_{0}}\right\rangle=e^{-i \varphi}\left\langle\frac{d}{d \omega_{0}} \bar{a}\right|[K|\bar{a}\rangle]
$$

soit

$$
\left\langle\overline{\mathrm{a}} \mid \frac{\mathrm{d} \overline{\mathrm{b}}}{\mathrm{d} \omega_{0}}\right\rangle=\left\langle\frac{\mathrm{d} \overline{\mathrm{a}}}{\mathrm{d} \omega_{0}} \mid \overline{\mathrm{b}}\right\rangle .
$$

D'autre part $\langle\overline{\mathrm{a}} \mid \overline{\mathrm{b}}\rangle$ étant identiquement nul, on obtient en décrivant $\langle\overline{\mathrm{a}} \mid \overline{\mathrm{b}}\rangle$ par rapport à $\omega_{0}$

$$
\left\langle\frac{\mathrm{d} \overline{\mathrm{a}}}{\mathrm{d} \omega_{0}} \mid \overline{\mathrm{b}}\right\rangle+\left\langle\overline{\mathrm{a}} \mid \frac{\mathrm{d} \overline{\mathrm{b}}}{\mathrm{d} \omega_{0}}\right\rangle=0 .
$$

En comparant (A.22) et (A.23), on en déduit :

$$
\left\langle\overline{\mathrm{a}} \mid \frac{\mathrm{d} \overline{\mathrm{b}}}{\mathrm{d} \omega_{0}}\right\rangle=0 \text {. }
$$

\section{Bibliographie}

[1] On trouve les références des nombreux travaux originaux sur ces sujets dans les articles suivants :

Brossel, J., Pompage optique, Optique et Electronique Quantique (Gordon and Breach) les Houches 1964.

Cohen-TANnoudj, C., Cargèse lectures in physics, Vol. 2 (Gordon and Breach) 1968.

Putlitz, Zu, Double resonance and level crossing spectroscopy, Proceedings of the 1st. International Conference on Atomie Physics (Plenum Press, New York) 1969.
[2] LANDAU, L., Lifchitz, E., Mécanique Quantique, Théorie non relativiste (Edition Mir, Moscou) 1967 chap. XI, § 79.

[3] Haroche, S., Thèse, Paris (1971), Ann. de Phys. 6 (1971) 189 et 327.

[4] Kato, T., J. Phys. Soc. Jap. 5 (1950) 435.

[5] Messiah, A., Mécanique quantique Tome II (Dunod) 1964.

[6] Grynberg, G., Thèse de $3^{e}$ cycle, Paris, 1972. 